\title{
Influence of vmPFC on dmPFC Predicts Valence-Guided Belief Formation
}

\author{
[Dojana Kuzmanovic, ${ }^{1}$ - Lionel Rigoux, ${ }^{1,2}$ and ${ }^{\circledR}$ Marc Tittgemeyer ${ }^{1}$ \\ ${ }^{1}$ Translational Neurocircuitry Group, Max Planck Institute for Metabolism Research Cologne, 50931 Cologne, Germany, and ${ }^{2}$ Translational Neuromodeling \\ Unit, Institute for Biomedical Engineering, University of Zurich and ETH Zurich, 8032 Zurich, Switzerland
}

When updating beliefs about their future prospects, people tend to disregard bad news. By combining fMRI with computational and dynamic causal modeling, we identified neurocircuitry mechanisms underlying this optimism bias to test for valence-guided belief formation. In each trial of the fMRI task, participants $(n=24,10$ male) estimated the base rate (eBR) and their risks of experiencing negative future events, were confronted with the actual BR, and finally had the opportunity to update their initial self-related risk estimate. We demonstrated an optimism bias by revealing greater belief updates in response to good over bad news (i.e., learning that the actual BR is lower or higher than expected) while controlling for confounds (estimation error and personal relevance of the new information). Updating was favorable when the final belief about risks improved (or at least did not worsen) relative to the initial risk estimate. This valence of updating was encoded by the ventromedial prefrontal cortex (vmPFC) associated with the valuation of rewards. Within the updating circuit, the vmPFC filtered the incoming signal in a valence-dependent manner and influenced the dorsomedial prefrontal cortex (dmPFC). Both the valence-encoding activity in the vmPFC and its influence on the dmPFC predicted individual magnitudes of the optimism bias. Our results indicate that updating was biased by the motivation to maximize desirable beliefs, mediated by the influence of the valuation system on further cognitive processing. Therefore, although it provides the very basis for human reasoning, belief formation is essentially distorted to promote desired conclusions.

Key words: belief update; computational modeling; DCM; optimism bias; value; vmPFC

\section{Significance Statement}

The question of whether human reasoning is biased by desires and goals is crucial for everyday social, professional, and economic decisions. How much our belief formation is influenced by what we want to believe is, however, still debated. Our study confirms that belief updates are indeed optimistically biased. Critically, the bias depends on the recruitment of the brain valuation system and the influence of this system on neural regions involved in reasoning. These neurocircuit interactions support the notion that the motivation to maximize pleasant beliefs reinforces those cognitive processes that are most likely to yield the desired conclusion.

\section{Introduction}

Not only extrinsic rewards such as tasty food, but also internal processes such as desirable beliefs or positive emotions, are expected to evoke pleasant states. Believing that one is attractive and intelligent (Eil and Rao, 2011; Korn et al., 2012) or that one's future will be bright (Sharot et al., 2011) has a positive subjective

\footnotetext{
Received Jan. 30, 2018; revised July 17, 2018; accepted July 20, 2018.

Author contributions: B.K. wrote the first draft of the paper; B.K., L.R., and M.T. edited the paper; B.K. designed research; B.K. performed research; B.K. and L.R. analyzed data; B.K., L.R., and M.T. wrote the paper.

We thank Morné Truter for proofreading and valuable comments on an earlier draft of the manuscript and Thorben Huelsduenker for assistance with data collection.

The authors declare no competing financial interests.

Correspondence should be addressed to Bojana Kuzmanovic, Max Planck Institute for Metabolism Research, Translational Neurocircuitry Group, Gleuelerstr. 50, 50931 Cologne, Germany. E-mail: bojana.kuzmanovic@sf.mpg.de. DOI:10.1523/JNEUROSCI.0266-18.2018

Copyright $\odot 2018$ the authors $\quad 0270-6474 / 18 / 387996-15 \$ 15.00 / 0$
}

value, which is why people tend to be motivated to maintain such beliefs (Sharot and Garrett, 2016). In turn, the motivation to maximize pleasant beliefs has been hypothesized to reinforce those cognitive processes that are most likely to yield a desired conclusion (Kunda, 1990; Hughes and Zaki, 2015).

However, motivational influences on reasoning have been controversially debated (Kunda, 1990; Shah et al., 2016; Garrett and Sharot, 2017; Kuzmanovic and Rigoux, 2017). How can we prove whether specific conclusions are reinforced by desires when these processes are hidden from direct observation and can operate outside of awareness (Tesser, 2000)? One way is to identify systematic, valence-dependent biases in information integration. For instance, when participants were given new information relevant to their current belief, they were more likely to incorporate good than bad news (e.g., indicating a lower versus higher 
risk than initially expected) to update their belief (Sharot et al., 2011; Kuzmanovic et al., 2016a). Circumventing self-report, such asymmetric updating provides an individual index of the optimism bias by exploiting actual belief formation behavior.

Another difficulty is that no reward has a fix subjective value, neither the extrinsic nor the intrinsic ones. Tasty food, for instance, is more pleasant during a hungry state. This is directly reflected in the activity of brain regions encoding the reward value such as the ventromedial prefrontal cortex (vmPFC) (Bartra et al., 2013; Chase et al., 2015): neurons in the vmPFC that fired in response to tasty food during a hungry state no longer showed this response after satiety (Grabenhorst and Rolls, 2011). Likewise, desirable beliefs may be more pleasant after threatening a person's self-worth (Roese and Olson, 2007; Rudman et al., 2007). Indeed, the magnitude of the optimism bias substantially varied across the participants (Sharot et al., 2011; Kuzmanovic and Rigoux, 2017). We assume that the belief updating should be biased only in the subjects who indeed assign a positive value to avoiding threatening and enhancing desirable beliefs. This allows us to infer the current value of a specific reward (favorable beliefs) from the reinforcement of the behavior leading to this reward (updating biased toward favorable beliefs).

The present study aims to demonstrate that desirable beliefs have an incentive salience and therefore can guide updates by influencing ongoing cognitive processing. To this end, neural circuits of belief updating were identified by using an established fMRI paradigm. Recently (Kuzmanovic et al., 2016a), we have shown that the vmPFC encoded the positive value of favorable self-related (but nor other-related) belief updates, indicating that the brain transforms beliefs into the same common value scale as classical rewards. Further, we isolated cognitive components and formally controlled for confounds using computational modeling to validate conclusions about valence-dependent update behavior (Kuzmanovic and Rigoux, 2017). Based on this previous work and the central role of the vmPFC in value encoding (Bartra et al., 2013; Chase et al., 2015), we hypothesized that the positive value of favorable updating would be encoded by the vmPFC. Moreover, we expected that only those individuals who showed an optimism bias would have a strong neural response to favorable updating. Finally, we used dynamic causal modeling to investigate mechanisms underlying valence-dependent updating. We hypothesized that the contexts of favorable and unfavorable updating would modulate coupling among regions recruited during updating and that the identified valuation system would influence other regions involved in belief formation. Our results provide evidence for a motivationally biased belief formation that is mediated by the value encoding in the vmPFC and the influence of the vmPFC on the dorsomedial PFC (dmPFC).

\section{Materials and Methods \\ Participants}

Forty subjects were recruited from the institute's subject database. A total of four participants were excluded because of problems with task performance. Two participants recognized that the base rates (BRs) were manipulated and one individual did not update estimates in $84 \%$ of trials ( mean of the included sample $=30.09 \%, \mathrm{SD}=16.13$; exclusion threshold $=66.67 \%$ ). Last, one participant updated estimates away from the presented BR in $18 \%$ of trials, indicating problems with task understanding (mean of the included sample $=2.66 \%, \mathrm{SD}=4.12$; exclusion threshold $=15 \%$ ). Data from another 12 participants were excluded due to excessive head movement in the MR scanner that exceeded a threshold of 1.5 framewise displacement (Power et al., 2012). This was necessary to account for increased sensitivity to motion-related artifacts in multiband acquisition for functional imaging (see below for acquisition parame- ters). Therefore, in total, 24 subjects were included into the analysis (10 male, mean age $=27.38, \mathrm{SD}=5.15$ ). Adding the 12 subjects with excessive motion to the sample of 24 subjects revealed the same behavioral results (see "Task performance" section in the Results).

\section{Experimental design}

The experiment was conducted during the acquisition of fMRI scans using Presentation 18.1 (Neurobehavioral Systems) and consisted of 80 trials with 80 different adverse life events (e.g., cancer or car theft). Participants began each trial with estimating the BR of an adverse life event (eBR) (see Fig. 1). Next, they were asked to estimate their own likelihood of experiencing the life event in their lifetime (first estimate, E1) and were subsequently presented with the actual BR. Subjects were instructed that the $\mathrm{BR}$ refers to the probability of the respective event occurring to persons of the same sex and age, living in the same sociocultural environment, as determined by the German Federal Statistical Office (Statistisches Bundesamt). At the end of each trial, participants had to reestimate their own risk (second estimate, E2).

The critical behavioral measure was the size of the update, the difference between E1 and E2. Subjects were expected to update their first risk estimate after being confronted with a BR different from the one they initially assumed. This difference between $\mathrm{eBR}$ and $\mathrm{BR}$ indicated the estimation error $(\mathrm{EE})$ where $\mathrm{EE}=|\mathrm{eBR}-\mathrm{BR}|$. In half of the trials, $\mathrm{BR}$ was desirable (better than expected, i.e., eBR $>$ BR; good news, GOOD), and in the other half, $\mathrm{BR}$ was undesirable (worse than expected, i.e., eBR $<$ $\mathrm{BR}$; bad news, BAD). We expected participants to change their risk estimates on average toward the new information. That is, upon an actual BR that is lower than expected, participants should decrease their risk estimates. Conversely, upon an actual BR that is higher than expected, participants should increase their risk estimates. Indeed, updates toward the direction opposed to the new information were very rare $(M=2.66 \%$, $\mathrm{SD}=4.12$ ). This is also reflected in the desirability-dependent computation of updates that ensures that positive values indicate an update toward the new information equally for GOOD and BAD (see Table 1). Valencedependent bias in updating was present when GOOD and BAD trials

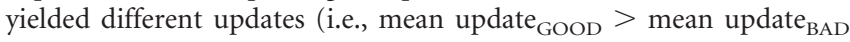
indicates an optimism bias).

Participants were free to report a probability anywhere between $1 \%$ and $99 \%$. Starting from $50 \%$ in eBR, they selected the desired probability by using two buttons to increase or decrease the number displayed on the screen (see Fig. 1, green font in eBR, E1, and E2) and a third button to confirm the selected choice. Subjects were instructed to use both hands. In the first half of the experiment, they used the right hand for selecting the percentage number and the left hand for confirming it and, in the second half, the other way around (order counterbalanced across subjects). In E1, the starting number equaled the one selected in eBR and, in $\mathrm{E} 2$, the starting number corresponded to the one selected in E1.

For eBR, E1, and E2, the response display was activated after a $2 \mathrm{~s}$ interval. Subjects were instructed to use the first $2 \mathrm{~s}$ to think about their estimate and then had a maximum of $10 \mathrm{~s}$ to respond (see Table 1 for mean reaction times). BR was presented for $2 \mathrm{~s}$. The intervals within and between the trials consisted of a fixation cross and were jittered (Mumford et al., 2015): the three interstimulus intervals within the trial (between eBR and E1, E1 and BR, and BR and E2) ranged between 2375 and $4625 \mathrm{~ms}$, with a mean of $3500 \mathrm{~ms}$, and the intertrial intervals ranged between 4875 and $7125 \mathrm{~ms}$, with a mean of $6000 \mathrm{~ms}$. The average task duration was $48 \min (\mathrm{SD}=2.45)$.

GOOD and BAD trials were rendered comparable with respect to the following: (1) number of trials, (2) mean size of EE, and (3) range of actual BRs. Furthermore, the assignment of stimuli to the two conditions (GOOD and BAD), the different EE sizes, and the order of trials were randomized anew for each subject. This was accomplished by manipulating the $\mathrm{BR}$ unbeknownst to subjects. To generate a desirable BR, a number between 1 and 25 was subtracted from the eBR and, to generate an undesirable BR, a number between 1 and 25 was added to the eBR. In addition, BRs were capped between $1 \%$ and $90 \%$ because BRs exceeding this range are likely to appear implausible.

However, this manipulation of BR was sometimes constrained by subjects' responses. For instance, when the eBR was close to or above $90 \%$, 
trials that were scheduled to generate bad news could not be realized (e.g., when eBR was $90 \%$, no greater BR could be generated because BRs were capped between $1 \%$ and $90 \%$ ). Instead, a number lower than $90 \%$ was presented (a random number between $85 \%$ and $90 \%$ ), generating good news. This reversal of the scheduled trial valence $(\mathrm{M}=1.30, \mathrm{SD}=2.20)$ can be made responsible for the condition-wise differences in number of trials (the reversal occurred only in BAD trials, thereby decreasing the number of realized BAD trials and increasing the number of realized GOOD trials) and eBR and E1 (only BAD trials with high eBRs were possible candidates for such a reversal and $\mathrm{eBR}$ and $\mathrm{E} 1$ were highly correlated, as one would expect, see Fig. 2E,F). Supporting this assumption, number of trials and eBR differed between the conditions only in subjects with reversals (and/or $\mathrm{EE}=0 ; t_{(11)}=2.68, p=0.022$, $\mathrm{eBR}, t_{(11)}=6.86$, $p<0.001)$, but not in subjects without such irregularities $\left(t_{(11)}=0.32\right.$, $p=0.755$, eBR, $\left.t_{(11)}=2.06, p=0.064\right)$. We nevertheless achieved satisfactory balanced distributions of number of trials, eBR, E1, and EE between conditions (e.g., on average 78.17 of 80 trials could be realized, and the mean difference between GOOD and BAD was 1.75 trials; see Table 1). Additional details on the experimental design and the BR manipulation algorithm have been described previously (Kuzmanovic and Rigoux, 2017).

Before the experiment, all participants received written instructions and completed six practice trials with stimulus events not used in the experiment. In a final debriefing after the experiment, a funneled procedure was used to ensure that subjects did not suspect the manipulation of the BRs or the purpose of the study. All procedures were in accordance with the World Medical Association Declaration of Helsinki and were approved by the local ethics committee of the Medical Faculty of the University of Cologne, Germany (15-255).

\section{Acquisition parameters}

The MRI data were acquired by using a Magnetom Trio Prisma ${ }^{\text {fit }}$ 3T whole-body scanner and a 64-channel head coil (Siemens AG Medical Solutions). During the update experiment, fMRI data were acquired in one session with a slice accelerated multiband echoplanar imaging sequence (Xu et al., 2013) covering the whole brain ( $\mathrm{TR}=1050 \mathrm{~ms}$, $\mathrm{TE}=$ $37.40 \mathrm{~ms}$, field of view $=212 \times 212 \times 144 \mathrm{~mm}^{3}$, voxel size $=2 \times 2 \times 2$ $\mathrm{mm}^{3}, 72$ oblique axial slices, multiband acceleration factor 6 ). In addition, we acquired two images with reversed phase encoding directions (anterior-posterior or posterior-anterior) for the purpose of estimating and correcting the susceptibility-induced distortion using topup (TR $8240 \mathrm{~ms}$, TE $69 \mathrm{~ms}$, field of view $212 \times 212 \times 144 \mathrm{~mm}^{3}$, voxel size $2 \times$ $2 \times 2 \mathrm{~mm}^{3}, 72$ oblique axial slices). High-resolution T1-weighted images were obtained from the institute's subject database (MDEFT, TR 1930 $\mathrm{ms}$, TE $5.80 \mathrm{~ms}$, field of view $256 \times 256 \times 160 \mathrm{~mm}^{3}$, voxel size $1 \times 1 \times$ $1.25 \mathrm{~mm}^{3}, 128$ sagittal slices, or MPRAGE, TR $2300 \mathrm{~ms}$, TE $2.32 \mathrm{~ms}$, field of view $256 \times 256 \times 192 \mathrm{~mm}^{3}$, voxel size $0.9 \times 0.9 \times 0.9 \mathrm{~mm}^{3}, 213$ sagittal slices).

\section{Statistical analyses}

\section{Analysis of task performance}

Before analyses, the following trials were excluded: trials with missing responses $(\mathrm{M}=0.83, \mathrm{SD}=1.01)$, trials with $\mathrm{EE}=0$ (e.g., when eBR was $1 \%$ in a GOOD trial, $\mathrm{BR}$ was also $1 \% ; \mathrm{M}=0.88, \mathrm{SD}=1.26$ ), and outliers (trials in which the update exceeded 4 SDs of the subjects' mean; $\mathrm{M}=$ 0.13 , SD $=0.34)$. For each subject, trials were divided into two conditions: good news (GOOD; BR < eBR) and bad news (BAD; BR > eBR). Optimism bias was assessed by comparing updates in GOOD trials with those in BAD trials (mean update ${ }_{\mathrm{GOOD}}-$ mean update $_{\mathrm{BAD}}$ ). Note that, on average, participants were expected to decrease their risk estimates after good news and to increase their risk estimates after bad news. Therefore, for both update $\mathrm{GOOD}_{\mathrm{GO}}$ and update $\mathrm{BAD}_{\mathrm{BAD}}$, positive values indicate an update toward the new information (see Table 1 for statistics of task variables; also see Fig. 2A). Furthermore, for each participant, we conducted a linear regression to predict his or her updates on each trial using valence of news (GOOD vs BAD) while including eBR, E1, and EE as covariates (all measures $z$-scored within subject). For repeated measures, the SD of the paired differences was used as a standardizer for Cohen's $d$ (Cumming, 2014).
In addition, we performed computational modeling of belief updating. The model-based approach allows to formally control for fluctuations in trialwise eBR, E1, and EE across conditions and to simulate unbiased updating based on observed trial-wise EE and personal relevance (PR). Building on previous work (Kuzmanovic and Rigoux, 2017), the model of belief updating was formalized as follows:

$$
\begin{gathered}
\text { Update }=L R^{*} E E^{*} P R, \\
L R_{G O O D}=\text { Alpha }+ \text { Asymmetry } \\
L R_{B A D}=\text { Alpha }- \text { Asymmetry }
\end{gathered}
$$

This model relies on the generic form of reinforcement learning, in which update is proportional to the EE (equivalent to prediction error). In addition, EE is weighted by the learning rate (LR), which indicates the general tendency of each subject to update their beliefs in response to the EE. To test for the optimism bias (asymmetric learning), LR was estimated separately for good and bad news (see also Palminteri et al., 2017; Lefebvre et al., 2017) and therefore has two components. The general component, alpha $(\alpha)$, indicates the tendency to learn from errors independently of the valence of news. $\alpha$ equal 1 indicates that update is exactly equal to $\mathrm{EE}$, while $\alpha$ smaller than 1 indicates updates smaller than EE. Asymmetry $(A)=0$ indicates equal learning rates for GOOD and BAD, whereas A different from zero indicates that the resulting learning rates systematically differ for GOOD and BAD (e.g., A > zero indicates lower learning rates and thus smaller updates for BAD than for GOOD).

EE is also weighted by the PR (corresponds to "relative personal knowledge" in Kuzmanovic and Rigoux, 2017). PR indicates the difference between eBR and E1 relative to the maximal possible difference in each trial (see Table 1 for the exact equation). Recently, we have demonstrated that the computational model of belief updating that weighted $\mathrm{EE}$ with PR was superior to the model without any consideration of PR (Kuzmanovic and Rigoux, 2017). This shows that the more people felt detached from the reference population, the more irrelevant BRs became for their updates of risk estimates (e.g., if I do not have a car, I will not consider the $\mathrm{BR}$ of car theft). $\mathrm{PR}$ ranged from 0 to 1 , with $\mathrm{PR}=1$ when a subject perceives her risk to be equal to those of the average person $(\mathrm{eBR}=\mathrm{E} 1$; see Fig. 1 for an example $)$ and $\mathrm{PR}=0$ when the perceived difference (eBR vs E1) is maximal. Therefore, EE weighted by PR indicates a subjective error (SE), where the impact of the EE on update is also determined by the PR of the new information.

Using the VBA toolbox (Daunizeau et al., 2014), we implemented competing models and tested which of these best accounted for the observed update behavior. To test whether $\alpha$ was different from 1 and whether A was different from 0 , we generated all possible variations of the update equation by switching the parameters $\alpha$ and $\mathrm{A}$ on (by letting the parameter free) or off (by fixing the parameter's prior variance to zero). Therefore, four models ( $\alpha \mathrm{A}, \alpha, \mathrm{A}, \varnothing ; \alpha$ and A indicate that the respective parameter was let free) were estimated for each subject. Note that, by setting A to 0 (i.e., models $\alpha$ and $\varnothing$ ), we specified the null hypothesis that learning is unbiased. In the alternative hypothesis (i.e., models $\alpha \mathrm{A}$ and A), A was estimated for each participant. Model estimations yielded a posterior distribution across the parameters and an approximation to the evidence of the model. The approximated model evidence reflects the goodness of fit penalized for the complexity. We used the free-energy approximation that has been shown to be superior to other approximations such as AIC or BIC (Penny, 2012). Model evidence of all subjects and all tested models was then entered in a random effects Bayesian model comparison. For each model, this procedure estimates the following: (1) the probability of each subject to be best described by the respective model (model attributions), (2) the frequency in the population (estimated model frequency, $E f$ ), and (3) the protected exceedance probability ( $p x p$ ), which is the probability that the model predominates in the population above and beyond chance (see Rigoux et al., 2014 for more details).

\section{fMRI analyses}

Before analysis, the first 10 volumes were discarded to allow for magnetic saturation. First, functional images were corrected for motion and dis- 
tortion using the FSL (version 5.0.9) tools MCFLIRT and topup (Andersson et al., 2003; Smith et al., 2004). All further analysis steps including DCM were conducted using SPM12 (Wellcome Trust Centre for Neuroimaging, London) implemented in MATLAB R2014b (The MathWorks). The T1 image was normalized to the Montreal Neurological Institute (MNI) reference space using the unified segmentation approach and the ensuing deformation parameters were applied to (previously coregistered) functional images. Finally, functional images were smoothed using an 8 full-width-half-maximum Gaussian kernel.

Statistical analyses were conducted in the framework of a general linear model (GLM). At the single-subject level, conditions were modeled using a boxcar reference vector convolved with the canonical hemodynamic response function and its time derivative. The following events were modeled on separate regressors: eBR, E1, BR, E2, responses, and rest. The duration of eBR, E1, BR, and E2 was always set to $2 \mathrm{~s}$, as for events with responses (all except of BR) the response display was activated only after $2 \mathrm{~s}$. Responses for all events were modeled on one regressor (duration from the onset of the response event to the confirmation button press, which was also the beginning of the next interstimulusinterval). The instruction to switch hands after the first half of trials and the excluded trials (missing responses, $\mathrm{EE}=0$, and outliers; see "Analysis of task performance" section), if present, were modeled on the "rest" regressor. Motion parameters and a matrix with motion outlier volumes (identified using the tool fsl_motion_outlier at a threshold of 4 SD of intensity differences between subsequent volumes; Power et al., 2012) were included as multiple regressors of no interest. Low-frequency signal drifts were filtered using a cutoff of $128 \mathrm{~s}$. At the group level, flexible factorial design and a significance threshold of $p<0.05$, FWE corrected at the peak level with an extent threshold of 20 voxels were used. For the covariate analyses, we applied the same statistical threshold, but a lower extent threshold of 10 voxels.

Error tracking. We identified brain regions that encoded the errors experienced during the BR event. At that time, subjects were confronted with a different actual BR than the one they have estimated (i.e., the difference between eBR and $B R$ ). To obtain the effects separately for GOOD and $\mathrm{BAD}$, we split the $\mathrm{BR}$ trials into $\mathrm{BR}_{\mathrm{GOOD}}$ and $\mathrm{BR}_{\mathrm{BAD}}$, and tested for parametric modulation $(\mathrm{PM})$ by subjective error $(\mathrm{SE}=\mathrm{EE} *$ PR, see "Analysis of task performance" section). We focused on this subjective error processing because it was more relevant for the subsequent belief updating than the general error (i.e., EE; Kuzmanovic and Rigoux, 2017). The resulting nine regressors (eBR, E1, $\mathrm{BR}_{\mathrm{GOOD}}, \mathrm{PM}_{-} \mathrm{error}_{\mathrm{GOOD}}$, $\mathrm{BR}_{\mathrm{BAD}}, \mathrm{PM} \_$error ${ }_{\mathrm{BAD}}, \mathrm{E} 2$, responses, and rest) were only weakly correlated ( $\bar{r}$ averaged across subjects were between -0.29 and 0.14 ), indicating efficient parameter estimation. At the single-subject level, two contrast images were computed relative to the implicit baseline $\left(\mathrm{PM}_{-}\right.$error $_{\mathrm{GOOD}}$ and PM_error ${ }_{\mathrm{BAD}}$ ) and entered into group-level analysis. At the group level, we identified those regions that exhibited increasing or decreasing activation with increasing subjective error in both GOOD and BAD trials (global conjunction). Furthermore, we explored differences between PM_error ${ }_{\mathrm{GOOD}}$ and PM_error ${ }_{\mathrm{BAD}}$ and reported global conjunction results for significant results to clarify whether the difference related to different magnitudes of the same modulation effect (e.g., the positive correlation between BOLD and error was stronger in BAD than in GOOD) or to modulation effects of opposite direction (e.g., the correlation between BOLD and error was positive in BAD, but negative in GOOD). To be able to illustrate group effects of different sizes of error on the BOLD signal, we also computed a GLM that models three sizes of error (small, mid, and large) on three separate regressors separately for GOOD and BAD (see Fig. 3A, line chart). Small, mid, and large categories were generated by dividing the sorted array of values into three subarrays that did not share same values and were maximally similar with respect to the number of elements (this procedure was the same for errors and update'; see below). Finally, we tested whether the extent of error tracking correlated with the learning rate component $\alpha$ across subjects by conducting a covariate analysis with one contrast per subject (average effect of PM_error ${ }_{\mathrm{GOOD}}$ and PM_error $\left.{ }_{\mathrm{BAD}}\right)$.

Valence of updating. To identify brain regions that encoded the valence of updating, we focused on the E2 event because at that time subjects were deciding upon updating their initial belief. E2 trials were split into
$\mathrm{E} 2_{\mathrm{GOOD}}$ and $\mathrm{E} 2_{\mathrm{BAD}}$ trials so that effects could be examined separately for GOOD and BAD. According to the valence of updating schematically shown in Figure $3 B$ (gray box), we tested for the positive correlation between the BOLD-signal and update in GOOD trials and for the negative correlation in BAD trials. To identify these opposed effects, we applied parametric modulation of $\mathrm{E} 2_{\mathrm{GOOD}}$ and $\mathrm{E} 2_{\mathrm{BAD}}$, respectively, by update size. The advantage of the PM procedure is that it allowed us to adjust the effect of update for EE, PR, and other potential confounds (e.g., by including three orthogonalized parameters in the following order: PR, EE, update; Mumford et al., 2015). The resulting 13 regressors

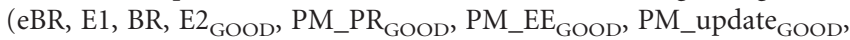

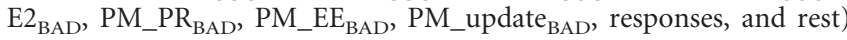
were only weakly correlated ( $\bar{r}$ averaged across subjects were between -0.29 and 0.03$)$, indicating efficient parameter estimation. The only exception was the negative correlation between BR and response $(\bar{r}=$ -0.49 ), which occurred because BR was the only event in the trial that was never associated with a subsequent motor response. Six contrast images were computed relative to the implicit baseline (PM_PR, PM_EE, and PM_update, separately for GOOD and BAD) and entered into group-level analysis. At the group level, we identified those regions that exhibited both increasing activation with increasing updates in GOOD trials, as well as increasing activation with decreasing updates in BAD trials, specified by the difference contrast (PM_update $\mathrm{GOOD}_{\mathrm{GO}}>\mathrm{PM}_{-}$update $\mathrm{BAD}_{\mathrm{BA}}$ ). In addition, we reported global conjunction results to clarify whether the difference related to different magnitudes of the same modulation effect (e.g., the positive correlation between BOLD and update was stronger in GOOD than in BAD) or to modulation effects of opposite direction (e.g., the correlation between BOLD and update was positive in GOOD, but negative in $\mathrm{BAD}$ ). Moreover, we tested whether the magnitude of the favorable updating effect correlated with the optimism bias across subjects by conducting a covariate analysis with one contrast per subject $\left(\right.$ PM_update $_{\mathrm{GOOD}}>$ PM_update $\left.{ }_{\mathrm{BAD}}\right)$.

Finally, we conducted two additional GLMs with categorical designs that split all trials into three sizes of update (small, mid, and large) separately for GOOD and BAD. To approximate the adjustment for $\mathrm{EE}$ within the modulation by update, we subtracted EE from update at each trial (update' $=$ update $-\mathrm{EE}$ ). That way, we controlled for the general effect that updates tend to be larger after larger EE, which may confound with the valence effect. Note that dividing update by EE would not be optimal because all trials with an update equal zero $(\mathrm{M}=30.09 \%, \mathrm{SD}=$ 16.13 ) would have yielded zero as well regardless of EE. This would not be appropriate because meaningful differences between zero updates in response to EEs of different sizes (e.g., $\mathrm{EE}=2$ and $\mathrm{EE}=20$ ) would have been concealed. For each subject, the numbers of trials across the three categories of updates were kept as similar as possible (numbers of trials did not differ; GOOD: $\mathrm{M}=13.32, \mathrm{SD}=0.85, F_{(2,69)}=0.58, p=0.560$; BAD: $\left.\mathrm{M}=12.74, \mathrm{SD}=1.02, F_{(2,69)}=0.09, p=0.913\right)$.

First, we used a GLM that modeled the three categories of update sizes separately for GOOD and BAD (six regressors) to illustrate group effects of different sizes of updates on the BOLD signal (see Fig. 3B, line chart). Second, we used another categorical GLM as a basis for the DCM analysis because the categorical levels can be more easily interpreted as inducing contextual modulatory effects in DCM than parametric variables (Stephan et al., 2010). According to the valence of updating schematically shown in Figure $3 B$ (gray box), this GLM collapsed the different update sizes into three valence categories corresponding to unfavorable (smallGOOD and large $\mathrm{BAD}_{\mathrm{BA}}$ updates, $\mathrm{U}$ ), mid (mid updates, $\mathrm{M}$ ), and favorable (large $_{\mathrm{GOOD}}$ and small $\mathrm{BAD}_{\mathrm{D}}$ updates, F) updating (Fig. $3 C$ ). Three contrast images were computed relative to the implicit baseline ( $\mathrm{E} 2_{\text {unfavorable }}$ $\mathrm{E} 2_{\text {mid }}$ and $\mathrm{E} 2_{\text {favorable }}$ ) and entered into group-level analysis. At the group level, we tested for brain regions that exhibited greater activation for favorable updates than for unfavorable updates $\left(E 2_{\text {favorable }}>\mathrm{E} 2_{\text {unfavorable }}\right)$. In addition, we identified those regions that were activated during updating independent of the valence (i.e., conjunction of all three levels of E2).

\section{DCM analyses}

DCM represents a hypothesis-led approach to understand neural circuits underlying observed brain responses (Friston, 2011). We used DCM to 


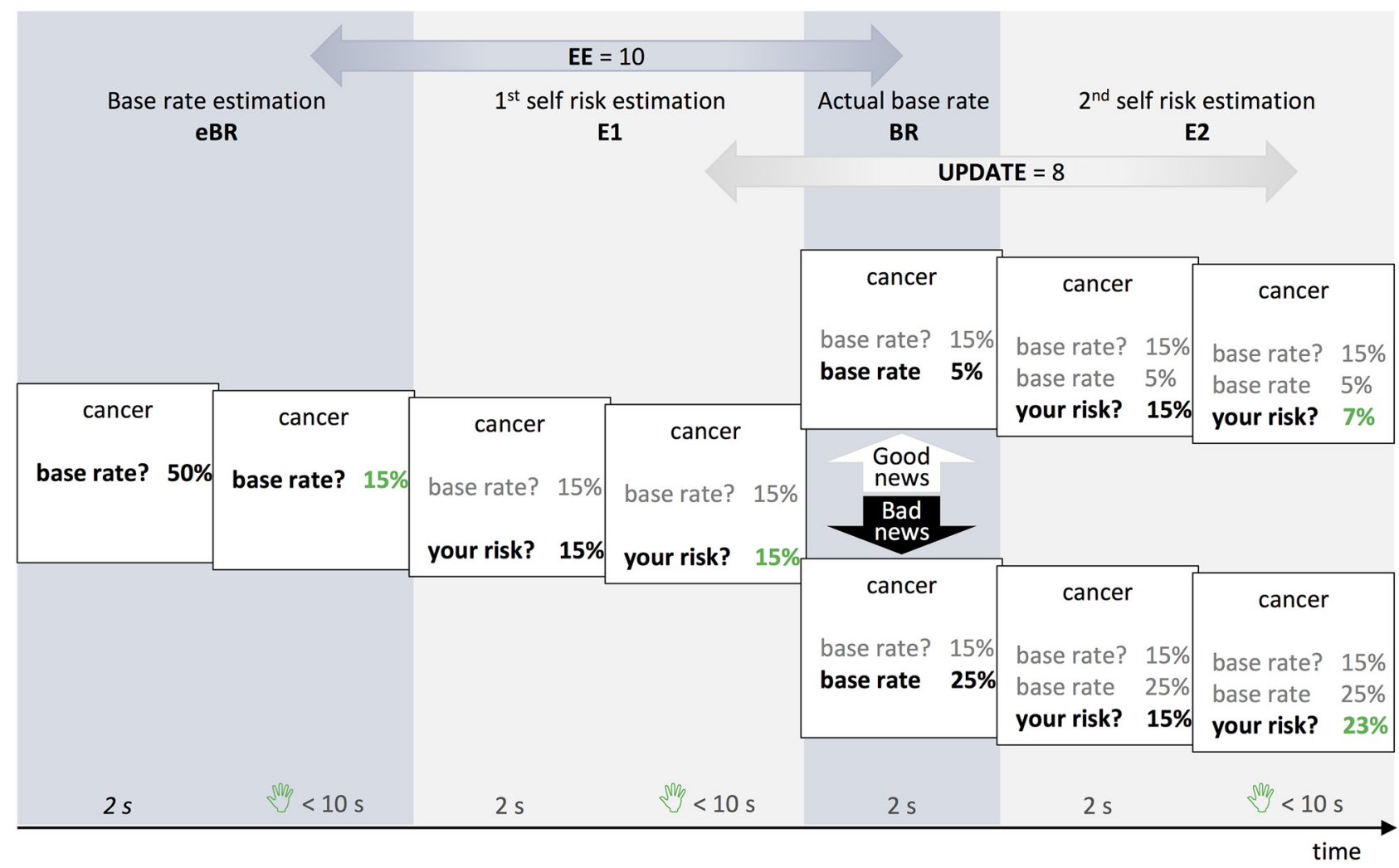

Figure 1. Outline and examples of experimental trials. Each experimental trial consisted of four succeeding events. With respect to a specific adverse life event (e.g., suffering from cancer), subjects had to estimate the BR (eBR) and their own risk (E1). They were then presented with the actual BR and had the opportunity to estimate their own risk again (E2). After identical eBR and E1, the upper progression of the hypothetical trial example shows a BR lower than expected indicating good news, whereas the lower progression shows a BR higher than expected indicating bad news. EEs corresponded to the difference between the eBR and the actual BR and the update corresponded to the difference between the first and the second self-risk estimate. Note that, in both trial examples, the EE is 10 and the update is 8 . For $\mathrm{eBR}$, E1, and E2, subjects were instructed to use response buttons to adjust the displayed number to match their estimate as soon as the number font changed to green (after $2 \mathrm{~s}$ ). Interstimulus intervals between eBR, E1, and E2, as well as intertrial intervals after E2, were jittered and consisted of a fixation cross (not shown here).

estimate and infer causal interactions among brain regions involved in belief updating (i.e., during the E2 event). To this end, competing models with different intrinsic coupling between regions and different taskdependent modulations of these couplings were specified. Each model corresponded to a specific hypothesis about how observed data were caused and Bayesian model selection was used to quantify the evidence for one model over another (Friston, 2011). Model inversion provided estimates of the model evidence and the corresponding effective connectivity. We tested whether the context of favorable and unfavorable updating modulated the coupling between distributed brain responses and whether value-coding regions exerted influence on other regions associated with cognitive processing.

First, we selected the nodes for the DCM based on the group results revealed by the simplified categorical GLM with three categories of valence of updates (unfavorable, mid, and favorable). The time series were extracted by computing the principal eigenvariate from 4-mm-diameter spheres (33 voxels) centered on the peak coordinates and adjusted for the effect of interest (F-contrast across the three categories of updates and the respective time derivatives).

Second, we specified competing models varying in their endogenous coupling and valence-dependent modulatory effects and inverted each model for every subject. Given that every brain region is connected reciprocally (Friston, 2011), the coupling in all models was cyclic; that is, all forward connections were accompanied by respective backward connections. We used a random-effects Bayesian model comparison to infer the optimal model structure by selecting the model with the best balance between accuracy and complexity.

Third, following the model selection, we performed a random-effects analysis of parameter estimates derived from the selected model using one-sample $t$ tests (Stephan et al., 2010). Additionally, we tested for correlations between parameter estimates and the optimism bias. For the sake of completeness, we report correlations between the two bias measures (optimism bias and A) and all model parameters in Table 4. Bonferroni correction was used to control for multiple comparisons: significance thresholds were adjusted for 7 tests for the matrix A parameters $(p<0.007)$ and 2 tests for the expected correlations $(p<0.025)$.

\section{Results}

Task performance

In the belief update task, subjects were asked to reconsider their risk estimates after being confronted with either good news (BRs of the risks were lower than initially expected) or bad news (BRs were higher than initially expected; Fig. 1). To assess valencebiased belief updating, we first tested whether subjects were more likely to take into account good news (GOOD) rather than bad news (BAD). Indeed, belief updates (the difference between the self-related risk estimates before and after being presented with the actual BR) following good news were significantly larger than the updates after bad news $\left(t_{(23)}=2.12, p=0.045\right.$, paired $t$ test, $d=0.43$; Fig. $2 A$, see Table 1 for the summary of all task variables). Furthermore, linear regression analyses revealed that updates were larger in GOOD than in BAD trials even after controlling for trial-wise $\mathrm{EE}\left(t_{(23)}=3.03, p=0.006, d=0.62\right.$, or for $\mathrm{eBR}, \mathrm{E} 1$, and $\mathrm{EE}, t_{(23)}=2.55, p=0.018, d=0.52$, one-sample $t$ tests). Therefore, these results indicate that belief updates were optimistically biased.

To implement an even more precise control for potential confounds and to further inform the fMRI analyses, we applied computational modeling. We tested whether the learning from actual BRs was asymmetric (different for GOOD and BAD, indicated by 
A

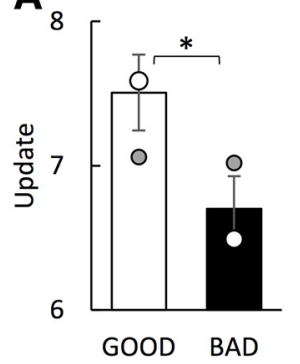

Simulated update $\bigcirc \alpha A \bigcirc \alpha$

D

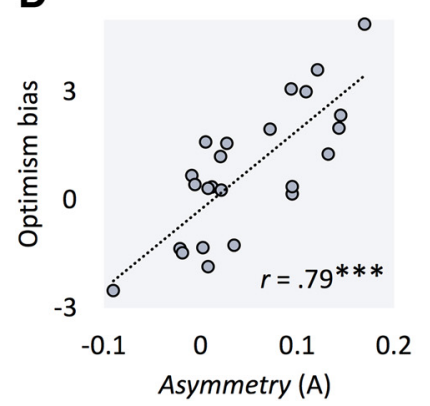

B

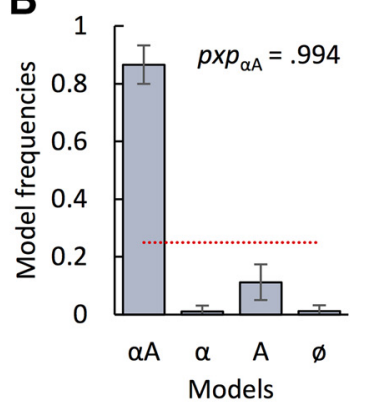

E

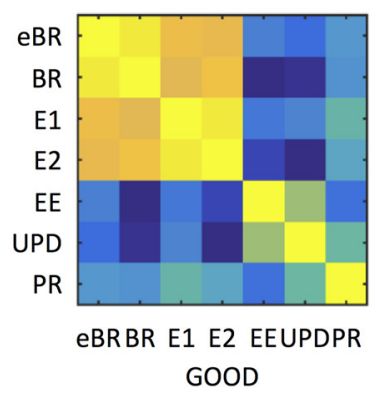

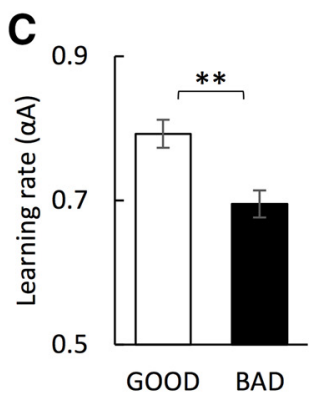

F
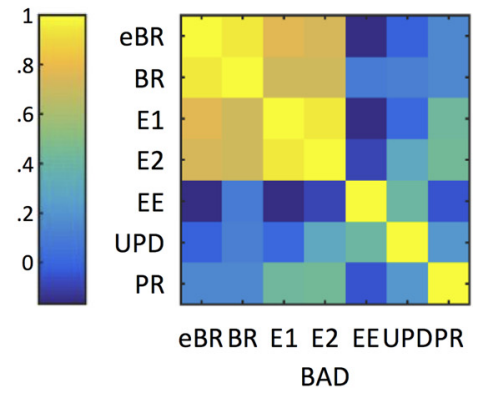

Figure 2. Task performance and computational modeling. $\boldsymbol{A}$, Bars show subjects' updates, that were significantly larger after good news (G00D) than after bad news (BAD). White dots represent simulations of updates by the "biased" computational model that assumes asymmetric learning rates for good and bad news ( $\alpha \mathrm{A}$, two free parameters, $\alpha$ and A). Gray dots indicate simulated updates resulting from the "unbiased" model that assumes identical learning rates for good and bad news $(\alpha$, one free parameter, $\alpha)$. The simulated unbiased updates provide a normative benchmark for rational updating with learning rates estimated for each subject under consideration of her or his exact trial history. Error bars indicate $S$ Es. $B$, Bayesian model comparison confirmed that the biased model $\alpha$ A best predicted subjects' updates. Model frequencies show that the majority of subjects were best described by the $\alpha A$ model above and beyond chance (red dashed line). Error bars indicate the posterior variance. $\boldsymbol{C}$, Learning rates extracted from the winning model $\alpha A$ were significantly higher after good than bad news. Error bars indicate $S E s$. $\boldsymbol{D}, 0$ ptimism bias (update $_{\mathrm{GOOD}}-$ update $_{\mathrm{BAD}}$ ) and $\mathrm{A}$ (estimated for each subject by the model $\alpha \mathrm{A}$ ) were significantly correlated (dots represent single subjects). $\boldsymbol{E}, \boldsymbol{F}, \mathbf{C o r r e l a t i o n s}$ between task variables separately for trials with good news $(\boldsymbol{E})$ and those with bad news $(\boldsymbol{F}) .{ }^{*} p<0.05,{ }^{* *} p<0.01$.

Table 1. Task variables

\begin{tabular}{|c|c|c|c|c|}
\hline \multirow[b]{2}{*}{ Parameter } & \multicolumn{2}{|l|}{$M(S D)$} & \multirow[b]{2}{*}{$p$} & \multirow[b]{2}{*}{ Source } \\
\hline & Good news & Bad news & & \\
\hline Number of trials & $39.96(1.45)$ & $38.21(2.38)$ & 0.024 & \\
\hline Estimated base rate (eBR) & $49.74(12.67)$ & $45.92(12.30)$ & 0.000 & Participants' response \\
\hline First estimate (E1) & $42.64(11.10)$ & $37.85(10.49)$ & 0.000 & Participants' response \\
\hline Presented base rate (BR) & $36.35(12.57)$ & $59.76(12.22)$ & 0.000 & Base rate algorithm \\
\hline Estimation error (EE) & $13.39(0.95)$ & $13.84(0.57)$ & 0.001 & $\mathrm{EE}=|\mathrm{eBR}-\mathrm{BR}|$ \\
\hline Second estimate (E2) & $35.12(10.86)$ & $44.55(11.26)$ & 0.000 & Participants' response \\
\hline Update & $7.51(2.58)$ & $6.70(2.20)$ & 0.045 & Update $_{\mathrm{GOOD}}=\mathrm{E} 1-\mathrm{E} 2$, Update $_{\mathrm{BAD}}=\mathrm{E} 2-\mathrm{E} 1$ \\
\hline Personal relevance (PR) & $0.70(0.12)$ & $0.69(0.13)$ & 0.287 & $\begin{array}{l}\text { for } E 1<e B R: P R=1-((e B R-E 1) /(e B R-1)) \\
\text { for } E 1>e B R: P R=1-((E 1-e B R) /(99-e B R)) \\
\text { for } E 1=e B R: P R=1\end{array}$ \\
\hline RT eBR (s) & $5.19(0.84)$ & $5.11(0.80)$ & 0.192 & Participants' response \\
\hline RTE1 (s) & $3.25(0.91)$ & $3.30(0.90)$ & 0.461 & Participants' response \\
\hline RT E2 (s) & $2.70(0.62)$ & $2.56(0.61)$ & 0.018 & Participants' response \\
\hline
\end{tabular}

All measures (except for number of trials) were recorded or computed for each trial and were then averaged separately for the conditions G00D and BAD and separately for each participant. Positive update values indicated updates toward the BR and negative values updates away from the BR ( $<3 \%$ of the trials). PR: 1 indicates equal risk perception for the average and oneself and 0 indicates maximally different risk perception for the average and oneself; note that PR corresponds to "relative personal knowledge" in Kuzmanovic and Rigoux, 2017. RT, Reaction time. $p$-values refer to paired two-tailed paired $t$ test with $n=24$.

the parameter A) while taking into account the EE, the PR of the new information, and the general tendency to learn from new information (learning rate component $\alpha$ ). Here, the EE was an important confound because larger errors generally tend to trigger larger updates. Also, when the new information is not regarded as personally relevant, updating of related beliefs tends to be reduced.

Bayesian model comparison of four competing models $(\alpha \mathrm{A}$, $\alpha, \mathrm{A}$, and $\varnothing$ ) provided additional support for the optimism bias. It revealed that the $\alpha \mathrm{A}$ model, which estimated both $\alpha$ and its $\mathrm{A}$ separately for each subject, predicted subjects' behavior significantly better than all other model versions ( $\alpha, \alpha$ fitted, A fixed to
0; A, $\alpha$ fixed to 1, A fitted; or $\varnothing, \alpha$ fixed to 1 and A fixed to 0$), E f=$ $0.87, p x p=0.994$ (Fig. $2 B$ ). A was significantly larger than zero $\left(\mathrm{M}=0.05, \mathrm{SD}=0.07, t_{(23)}=3.59, p=0.002\right.$, one-sample $t$ test, $d=0.73$ ), showing that participants' learning rates were higher in response to good news than to bad news $\left(\mathrm{LR}_{\mathrm{GOOD}}: \mathrm{M}=0.79\right.$, $\mathrm{SD}=0.19, \mathrm{LR}_{\mathrm{BAD}}: \mathrm{M}=0.70, \mathrm{SD}=0.18$; Fig. $\left.2 C\right)$. Furthermore, $\alpha$ was significantly smaller than $1\left(\mathrm{M}=0.74, \mathrm{SD}=0.18, t_{(23)}=\right.$ $-7.18, p<0.001$, one-sample $t$ test, $d=4.26$ ), showing that updates were on average smaller than the EEs. Finally, the optimism bias (derived from the observed task performance, mean update $_{\mathrm{GOOD}}-$ mean update $_{\mathrm{BAD}}$ ) and the A parameter (derived by the winning model $\alpha \mathrm{A}$ ) were significantly correlated $(r=0.79$, 
$p<0.001$; Fig. 2D). Although expected, the close relationship between these two bias measures also confirmed that the potential confounding variables (EE, PR) had no systematic influence in our task. Therefore, we can rule out that "seemingly optimistic updating" was induced by a differential consideration of EEs due to varying PR (Shah et al., 2016). Quite the contrary, the optimism bias was even stronger after taking EE and PR into account. Therefore, it is likely that earlier studies demonstrating the optimism bias, but lacking the enhanced experimental or formal computational control (Sharot et al., 2011, 2012; Garrett et al., 2014; Korn et al., 2014; Kuzmanovic et al., 2015, 2016a,b), are also not affected by these potential confounds. Furthermore, correlations between the different task variables, computed separately for trials with good news and bad news and then averaged across subjects (Fig. $2 E, F$ ), show that EE and updates correlated only very weakly with $\mathrm{eBR}, \mathrm{BR}, \mathrm{E} 1$, and $\mathrm{E} 2$ ( $\bar{r}$ ranging from -0.15 to 0.30 ). This is particularly important because it demonstrates that we succeeded in manipulating the desirability of EE independently of prior beliefs (i.e., the size of risk estimates eBR and E1). Furthermore, it shows that the valence of updates was independent of the size of the estimated risks (eBR, E1) or the presented BRs. Together, these findings provide a strong support for the notion that the difference in updating indeed reflected a valencedependent consideration of the new information.

Moreover, we assessed the updates that were simulated by the winning model $\alpha \mathrm{A}$ assuming asymmetric learning rates and by the unbiased model $\alpha$ given the trial-by-trial PR and EE. The updates simulated by the model $\alpha \mathrm{A}$ corresponded well to the actually observed updates $\left(\mathrm{M}_{\mathrm{GOOD}}=7.59, \mathrm{SD}=2.43 ; \mathrm{M}_{\mathrm{BAD}}=\right.$ $6.49, \mathrm{SD}=2.09$, see white dots in Fig. $2 A)$ and were larger in GOOD than in BAD trials $\left(t_{(23)}=3.85, p=0.001\right.$, paired $t$ test, $d=0.79$ ). In contrast, the updates simulated by the unbiased model $\alpha$ did not differ across GOOD and BAD trial $\left(\mathrm{M}_{\mathrm{GOOD}}=\right.$ $7.06, \mathrm{SD}=2.21 ; \mathrm{M}_{\mathrm{BAD}}=7.02, \mathrm{SD}=2.20, t_{(23)}=0.22, p=0.830$, paired $t$ test, $d=0.04$; see gray dots in Fig. $2 A$ ). This comparison proves that subjects' asymmetric updating represents a true bias attributable to the different valence of the new information (good and bad news) and cannot be explained by any variations of other trial-by-trial variables (i.e., PR or EE; Shah et al., 2016; Kuzmanovic and Rigoux, 2017).

Furthermore, we compared floor and ceiling effects across GOOD and BAD and showed that controlling for these effects even enhanced the optimism bias effect. Floor and ceiling effects could occur if the size of the possible update was limited by the response scale (probabilities from $1 \%$ to $99 \%$ ). For example, in a GOOD trial, given an $\mathrm{EE}=5$ (e.g., $\mathrm{eBR}=10 \%, \mathrm{BR}=5 \%$ ) and an $\mathrm{E} 1=3 \%$, a subject would have only a limited space on the response scale to make an update toward a lower risk estimate (from $\mathrm{E} 1=3 \%$ to the end of the response scale of $1 \%$ ). Critically, this possible update should be at least as large as the size of the EE to enable unconstrained updating. This is rather conservative because the general learning rate component $\alpha$ was significantly smaller than 1 and because EEs were also weighted by PR that ranged between 0 and 1 . To test for floor and ceiling effects, we computed the size of possible update relative to EE for each trial (updspace- $\mathrm{EE}_{\mathrm{GOOD}}=(\mathrm{E} 1-1)-\mathrm{EE}$; updspace- $\mathrm{EE}_{\mathrm{BAD}}=(99-$ E1) - EE). Updspace-EE was lower in GOOD than in BAD, $t_{(23)}=-4.50, p<0.001\left(\mathrm{M}_{\mathrm{GOOD}}=28.24, \mathrm{SD}=11.03 ; \mathrm{M}_{\mathrm{BAD}}=\right.$ $47.31, \mathrm{SD}=10.51)$. Furthermore, the number of constrained update spaces (updspace-EE $<0$ ) was higher in GOOD than in $\mathrm{BAD}, t_{(23)}=5.08, p<0.001\left(\mathrm{M}_{\mathrm{GOOD}}=6.17, \mathrm{SD}=5.45 ; \mathrm{M}_{\mathrm{BAD}}=\right.$ $0.58, \mathrm{SD}=0.77)$. Repeating the analyses after excluding the trials with a constrained update space revealed an even stronger opti- mism bias effect $\left(t_{(23)}=3.54, p=0.002\right.$, paired $t$ test, $d=0.72$, $\mathrm{M}_{\mathrm{GOOD}}=8.20, \mathrm{SD}=2.64 ; \mathrm{M}_{\mathrm{BAD}}=6.80, \mathrm{SD}=2.23$ ), also when controlling for trialwise $\mathrm{EE}\left(t_{(23)}=4.67, p<0.001, d=0.95\right.$, or for eBR, E1 and EE, $t_{(23)}=4.10, p<0.001, d=0.84$, one-sample $t$ tests). Computational modeling analyses were not affected by the exclusion of trials with constrained updating due to formal consideration of the PR: A derived from the $\alpha$ A model $(E f=0.86$, $p x p=0.991)$ was significantly larger than zero $\left(t_{(35)}=3.50, p=\right.$ 0.002 , one-sample $t$ test, $d=0.72$ ). Together, these tests show that the optimism bias effect was even underestimated because of greater floor effects in GOOD trials.

We also examined the behavioral results after adding the 12 subjects with excessive motion to the sample of 24 subjects. These analyses yielded the same results as those with $n=24$. Belief updates following good news were significantly larger than the updates after bad news $\left(t_{(35)}=3.18, p=0.003\right.$, paired $t$ test, $d=$ $0.53)$ and A was significantly larger than zero $\left(t_{(35)}=4.35, p<\right.$ 0.001 , one-sample $t$ test, $d=0.73$ ). Finally, the postexperimental debriefing revealed that none of the included subjects suspected that the purpose of the task was to assess difference in belief updating depending on the valence of the new information. At the end of the debriefing, we carefully explained the purpose of the study as well as the manipulation of the BRs in a standardized written form. Following this information, only one subject reported that he was aware of the good news-bad news effect during the own task performance. Furthermore, two subjects reported that they had no concerns with respect to the presented BRs during the task, the majority (17) reported that they were surprised by some of the presented BRs, but did not doubt their validity, five subjects doubted that single surprising BRs were really valid and none of the included subjects reported having realized that the BRs were manipulated.

\section{fMRI results}

\section{Error tracking during $B R$}

Updating beliefs about self-related risks was triggered by erroneous expectations regarding the respective BRs. To investigate this crucial process, we identified brain regions that tracked the errors experienced upon the presentation of the actual BRs. PM analysis revealed that error tracking recruited the anterior cingulate cortex (ACC), the inferior frontal gyrus (IFG), the anterior insula, the middle orbital gyrus, and the dorsolateral prefrontal cortex (dlPFC; Fig. 3A, Table 2, contrast 1b). In these regions, the activity increased with decreasing error size (negative correlation between BOLD and error) for both conditions GOOD and BAD (for an example, see the line chart in Fig. $3 A$ for the average activity in dlPFC across three sizes of error). This negative correlation seems unexpected because brain regions such as the ACC and the anterior insula have been associated with error processing, novelty, and task difficulty (Wessel et al., 2012; Klein et al., 2013; Shenhav et al., 2014; Kolling et al., 2016; Bastin et al., 2017; Fouragnan et al., 2017) and thus were expected to increase activity with increasing error size. However, a seminal study on belief updating has also demonstrated negative correlation between EE size and activity in the IFG, which was moreover predictive of trait optimism (Sharot et al., 2011). Therefore, it may be necessary to reconsider the meaning of different outcomes in the specific context of the present experiment because contexts determine the reference point for values of options (Palminteri et al., 2015). Subjects' task and their "default option" was to revisit their prior beliefs due to new challenging information. Therefore, in the majority of trials, subjects indeed were confronted with BRs that markedly differed from what they expected and they 


\section{A Error tracking}
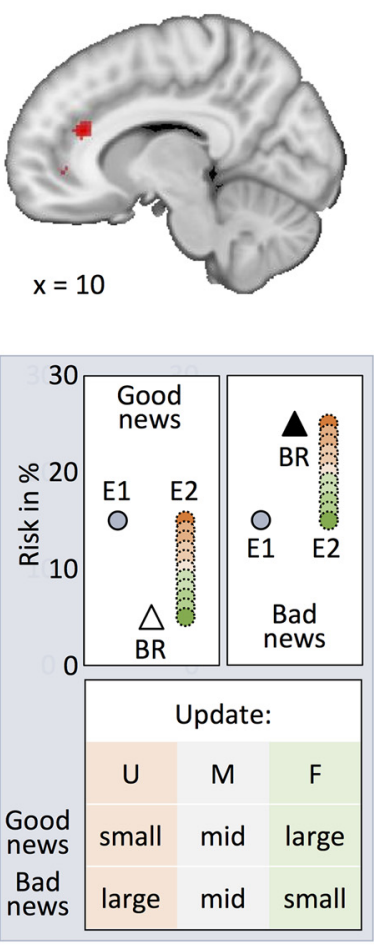

\section{General updating}

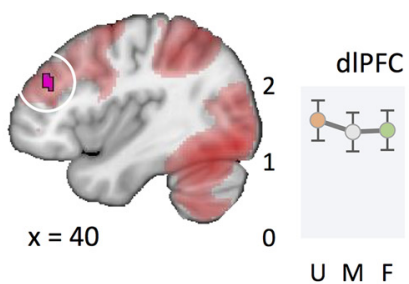

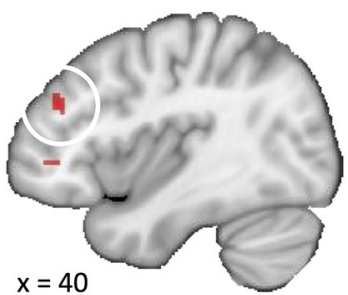

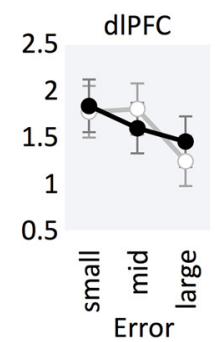

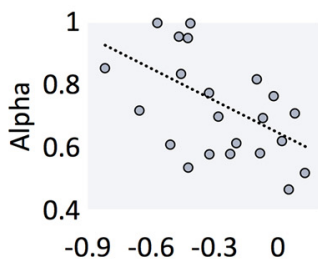

Effect in dIPFC

\section{B The valence of updating}
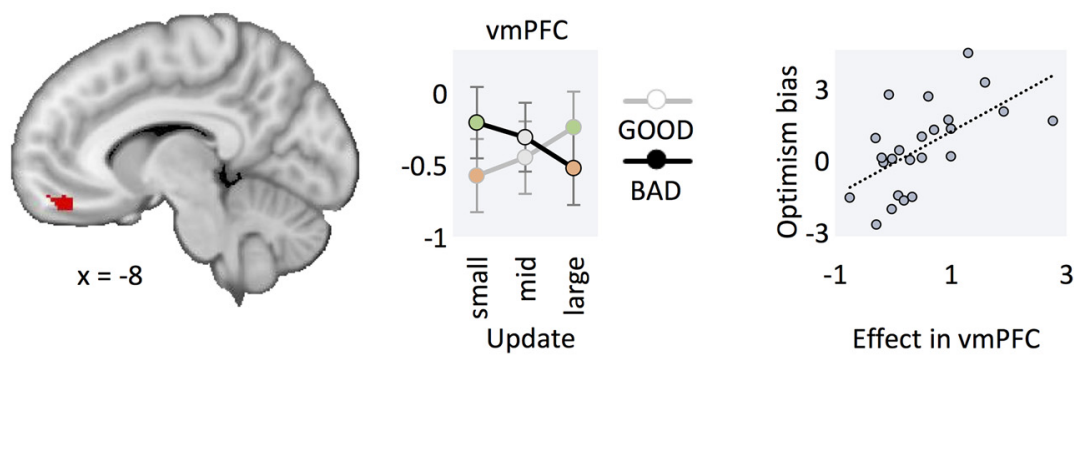

\section{Three categories of updates}

\footnotetext{
U, Unfavorable updates $\square$ F, Favorable updates; $\square p_{\text {FWE-corr. }}<.05$, peak level, whole brain; $\square$ Error tracking effect
}

Figure 3. Brain regions encoding errors and the valence of belief updating. $A$, When being confronted with the actual BR, errors in BR estimation (weighted by the PR) were tracked by the ACC, the IFG, the anterior insula, the middle orbital gyrus and the dIPFC. The line chart shows that the activity in the dIPFC (representative of all clusters) increased with decreasing error size (parametric modulation by error, negative correlation). Of all the involved regions, only in the dIPFC did the magnitude of the error tracking correlate with the general learning rate component $\alpha$ (see scatter plot). Therefore, subjects with a stronger error tracking in the dIPFC also more strongly adjusted their initial beliefs in response to errors. $B$, During the second risk estimation, the activity in the vmPFC encoded the valence of updating, adjusted for EE and PR. The gray box schematically illustrates the opposed valences of increasing updates after good and bad news (in this example, eBR $=E 1$ ). After good news, large updates are favorable because they ultimately change beliefs toward lower risk estimates and small updates are unfavorable because they let the opportunity to improve risk estimates pass by. In contrast, after bad news, large updates are unfavorable because they ultimately change beliefs toward higher risk estimates and small updates are favorable because they prevent worsening of risk estimates. Resulting valences are summarized in the table below: unfavorable (U), mid (M), and favorable (F) updates. The line chart shows that the activity in the vmPFC tracked the positive valence because it increased with increasing update sizes after good news but decreased with increasing update sizes after bad news. The scatter plot shows that subjects with a stronger optimism bias also demonstrated a greater tracking of favorable updating in the vmPFC. In $\boldsymbol{A}$ and $\boldsymbol{B}$, the line charts and the scatter plots were not used for statistical inference (which was performed in parametric modulation and covariate analyses within the SPM framework); they are shown solely for illustrative purposes. $C$, After demonstrating the valence effect with the more precise parametric modulation analysis presented in $\boldsymbol{B}$, a simplified analysis of updating was conducted as a basis for DCM. Here, all trials were assigned to three valence categories: those with unfavorable (U), mid (M), and favorable (F) updates (adjusted for EE). Conjunction across these three categories revealed a distributed network involved in general updating, overlapping with the error tracking effect in the dIPFC. Comparing trials with favorable and unfavorable updates revealed the differential recruitment of the vmPFC and the dmPFC during updating. The line charts show contrast estimates in the $\mathrm{IIPFC}, \mathrm{vmPFC}$, and dmPFC, respectively.

updated their belief. Relative to this, encountering trials with a small error increases the difficulty of the decision whether to update beliefs ("Is the actual BR different enough than expected, and is this difference relevant enough to drive an update of my own risk?"). Given the high accuracy of subjects' BR estimations in such trials, the alternative course of action to refrain from updating becomes increasingly valuable. We therefore speculate that increased activity in this network relates to enhanced initial comparison process that informs subsequent decisions about updating while maintaining behavioral flexibility (Kolling et al., 2016).

Furthermore, of all these error-tracking regions, only the activity in dIPFC correlated with the learning rate component $\alpha$ (covariate 


\begin{tabular}{|c|c|c|c|c|c|c|}
\hline & \multirow{2}{*}{$\begin{array}{l}\text { Cluster } \\
\text { size }\end{array}$} & \multicolumn{5}{|l|}{ Peak } \\
\hline & & $p_{\text {FWE-corr }}$ & $\mathrm{T}$ & $x$ & $y$ & Z \\
\hline \multicolumn{7}{|c|}{ 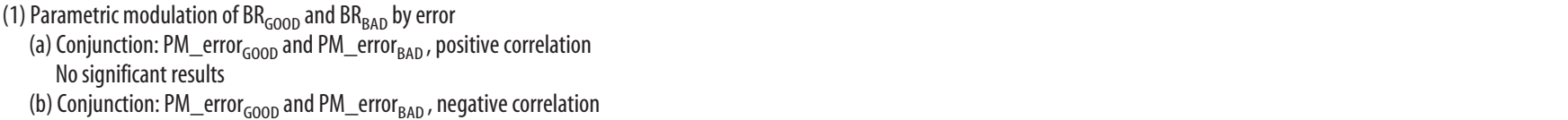 } \\
\hline Anterior cingulate cortex & 63 & 0.000 & 5.18 & 10 & 34 & 20 \\
\hline Inferior frontal gyrus (p. triangularis) & 45 & 0.001 & 4.09 & 46 & 44 & 0 \\
\hline Anterior insula & 37 & 0.001 & 4.05 & 28 & 22 & 6 \\
\hline Middle orbital gyrus & 30 & 0.002 & 4.00 & 16 & 50 & -2 \\
\hline dIPFCCOV_Alpha & 32 & 0.004 & 3.81 & 40 & 40 & 28 \\
\hline \multirow{2}{*}{\multicolumn{7}{|c|}{$\begin{array}{l}\text { (c) PM_error }{ }_{\mathrm{GOOD}}>\text { PM_error } \\
\text { No significant results } \\
\text { (d) } \mathrm{PM} \text { _error }{ }_{B A D}>\text { PM_error } \\
\text { GOOD }\end{array}$}} \\
\hline & & & & & & \\
\hline Cerebellum & 48 & 0.001 & 6.90 & -18 & -76 & -46 \\
\hline Middle occipital gyrus & 83 & 0.005 & 6.17 & -34 & -84 & 28 \\
\hline Superior parietal lobule & 47 & 0.006 & 6.15 & 24 & -56 & 48 \\
\hline $\begin{array}{l}\text { Conjunction: PM_error } \\
\text { Inferior occipital gyrus }\end{array}$ & 22 & 0.001 & 4.16 & 40 & -84 & -10 \\
\hline \multicolumn{7}{|c|}{ (2) Covariate analysis of error coding with alpha (masked with contrast 1b) } \\
\hline dIPFC & 12 & 0.008 & 4.46 & 40 & 38 & 30 \\
\hline
\end{tabular}

Error $=\mathrm{EE} * \mathrm{PR}$, based on the computational modeling of task performance. For significant differences between $\mathrm{PM}_{-}$error $_{\mathrm{GOOD}}$ and $\mathrm{PM} \_$error ${ }_{\mathrm{BAD}}$, we report global conjunction results to clarify whether the difference relates to different magnitudes of the same modulation effect (e.g., the positive correlation between BOLD and error was stronger in BAD than in GOOD) or to modulation effects of opposite direction (e.g., the correlation between BOLD and error was positive

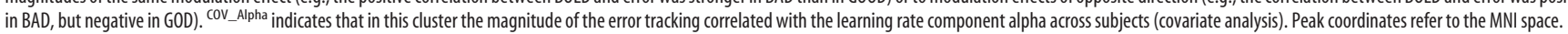

analysis masked with the conjunction contrast PM_error ${ }_{\mathrm{GOOD}}$ and PM_error ${ }_{\mathrm{BAD}}$, negative correlation; Table 2, contrast 2). Betas indicating the strength of the linear relationship between error and BOLD were extracted for each subject (PM analysis, at the peak [ 4038 30], averaged across PM_error ${ }_{\text {GOOD }}$ and PM_error$\mathrm{BAD}$ ) and plotted against $\alpha$ for illustrative purposes (scatter plot in Fig. $3 A$ ). Even when conducting the covariate analysis for the whole brain, an overlapping dlPFC cluster had the strongest correlation with $\alpha$ (peak at [38 3038], $\mathrm{T}=5.66,257$ voxels), albeit at a more liberal significance threshold ( $p<0.05$, FWE-corrected at the cluster level).

\section{Valence of updating}

The main aim of the fMRI analysis was to identify brain regions that encoded the valence of updating. The valence of updating was defined based on how much the second estimation resulted in either favorable or unfavorable risk estimates relative to the first estimation (see Fig. 3B, gray box, for an illustration). Note that, in GOOD trials, initial risk estimates were expected to decrease toward the actual BR that was lower than expected. Conversely, in $\mathrm{BAD}$ trials, risk estimates were expected to increase toward the actual BR that was higher than expected. Therefore, for GOOD trials, we assume that large updates would be experienced as favorable, because they result in lower final risk estimates. In contrast, for BAD trials, we assume that small (or zero) updates would be experienced as favorable, because they prevent an increase of final risk estimates. Therefore, we expected a positive correlation between the BOLD-signal and update in GOOD trials, and a negative correlation in BAD trials.

The parametric modulation analysis revealed that activity in the vmPFC had exactly this pattern (Fig. 3B, Table 3, contrast 1a), indicating that this region tracked favorable updating. The correlation between the BOLD signal in the vmPFC and update was greater in trials with good news than in trials with bad news (i.e., PM_update $\left.\mathrm{GOOD}_{\mathrm{G}}>\mathrm{PM}_{\text {_update }} \mathrm{BAD}\right)$. The conjunction contrast (i.e., PM_update $\mathrm{GOOD}_{\mathrm{GO}}$, positive correlation and PM_update $\mathrm{BAD}_{\mathrm{BAD}}$, negative correlation) confirmed that this effect implied contrary modulation effects for GOOD and BAD (positive correlation in GOOD and negative correlation in BAD; see the line chart in Fig.
$3 B)$. Moreover, the vmPFC was also the only area in the whole brain, in which the magnitude of this valence-tracking effect correlated with the optimism bias (Table 3 , contrasts $2 \mathrm{a}, 2 \mathrm{~b}$ ). This relationship to the task performance was illustrated by extracting betas indicating the strength of the differential linear relationship between update and BOLD for each subject (PM analysis, at the peak - $650-18$, PM_Error ${ }_{\mathrm{GOOD}}>$ PM_Error $_{\mathrm{BAD}}$ ) and plotting them against optimism bias (Fig. $3 B$, scatter plot).

Importantly, by including multiple orthogonalized parameters, we assessed variance that was uniquely explained by different update sizes above and beyond the effects of other relevant computational components of belief updating such as EE and PR (Mumford et al., 2015). Moreover, the valence-tracking effect in the vmPFC was significant even when we controlled for task variables other than EE and PR. Repeating the parametric modulation analysis while including BR as an additional regressor (four orthogonalized regressors: BR, PR, EE, update, separately for GOOD and BAD) yielded the involvement of the same vmPFC clusters for the three contrasts indicating the valence effect (Table 3 , contrast $1 \mathrm{a}$, including the conjunction, and contrast $2 \mathrm{~b}$; significance threshold as in the main analysis). In addition, including BR and E2 (five orthogonalized regressors: E2, BR, PR, EE, update, separately for GOOD and BAD) also confirmed the valencetracking effect in the vmPFC with respect to all three contrasts (albeit the contrast $2 \mathrm{~b}$ at a less stringent significance threshold of $p<0.001$, uncorrected, cluster size 216). Therefore, in contrast to previous studies investigating the rewarding effect of favorable new information per se (in the context of updating self-evaluations; Korn et al., 2012), the valence effect related to the relative improvement or worsening of initial beliefs, not to the valence of final beliefs (E2), the new information (BR), or to other variables (PR or EE).

Once we had demonstrated the effect of favorable updating while adjusting for BR, E2, EE, and PR using parametric modulation, we repeated the analysis with a simplified categorical model. Discrete levels of valence (e.g., unfavorable or favorable) can then be used in the following DCM analysis to specify contextual effects that modulate the intrinsic coupling within the 


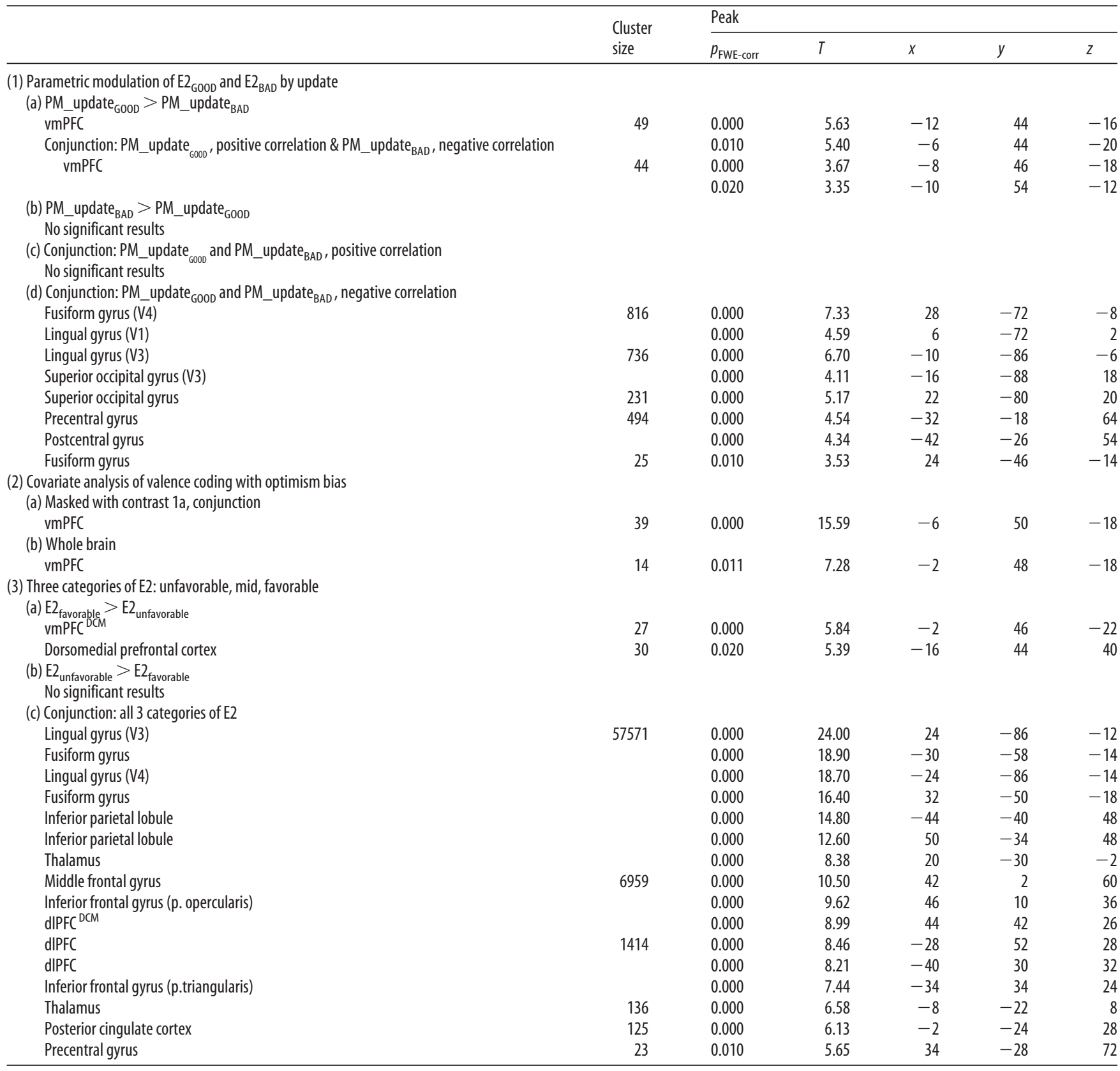

For significant differences between PM_update ${ }_{G O 0 D}$ and $P M \_$update ${ }_{B A D}$, we report global conjunction results to clarify whether the difference relates to different magnitudes of the same modulation effect (e.g., the positive correlation between BOLD and update was stronger in GOOD than in BAD), or to modulation effects of opposite direction (e.g., the correlation between BOLD and update was positive in G00D, but negative in BAD). Peak coordinates refer to the MNI space.

update circuit. According to the principle introduced above (and in the gray box in Fig. $3 B$ ), we specified three valence levels (Fig. $3 C)$ : unfavorable updating ( $\mathrm{U}$, small $\mathrm{GOOD}_{\mathrm{G}}$ and large $\mathrm{BAD}_{\mathrm{BA}}$ updates), mid updating ( $\mathrm{M}$, mid updates), and favorable updating ( $\mathrm{F}$, large $_{\mathrm{GOOD}}$ and small $\mathrm{BAD}_{\mathrm{B}}$ updates). As a result, we concatenated the valence of updates across trials with good and bad news. The simplified categorical analysis revealed similar results as the PM analysis, demonstrating greater activity in the vmPFC for favorable than for unfavorable updates (Fig. 3C, Table 3, contrast 3a). Moreover, the dmPFC showed a similar pattern of activity as the vmPFC. In addition, we tested for the conjunction effect across all three valence categories to identify brain regions that were generally activated during updating independently of valence. General updating revealed widespread activations including occipital, parietal, and frontal cortices (Fig. 3C, Table 3, contrast 3c).
Timing of updating

To determine whether the valence effect of updating did manifest already during the processing of $\mathrm{BR}$, we repeated the parametric modulation analysis (including PR, EE, and update), but defined the event BR instead of E2 as the unmodulated regressor. In this analysis, the contrast $\mathrm{PM} \_$update $\mathrm{GOOD}_{\mathrm{G}}>\mathrm{PM}_{\mathrm{P}}$ update $\mathrm{BAD}_{\mathrm{BA}}$ did not yield any significant effect even when using the vmPFC cluster as an inclusive mask at a less stringent significance level $(p<0.001$, uncorrected). This indicates that the encoding of the valence of belief updating by the vmPFC indeed occurred during the period of update consideration and not already during the reception of the new information.

This contradicts classical reinforcement tasks where belief updating is expected to occur upon a relevant outcome (e.g., if I choose the green and not the red square and win money, I update 
the value of choosing the green square immediately). However, there is a substantial qualitative difference between classical reinforcement tasks and the present task. Estimations of BRs of life events in a population recruit declarative memory to retrieve general knowledge and the feedback about the actual BRs indicates how accurate one was. Furthermore, estimating one's own risks of experiencing adverse events in the future represents a more complex cognitive process (including autobiographic and declarative memory) than deciding whether to choose a green or a red square in a gambling game. Therefore, it is plausible that subjects focused on their degree of accuracy at the time point of feedback and subsequently focused on the meaning of this new information for their own risk estimate.

Several arguments additionally support this notion. First, we explicitly instructed the subjects to reconsider their risk estimates during the "update phase" (first $2 \mathrm{~s}$ of E2, before the response buttons were activated; Fig. 1). Second, there was no need to memorize estimated and actual BRs (or first self-risk estimates) until the update phase because all preceding values were visible on the screen at all times (Fig. 1). Third, during the debriefing, a majority of subjects spontaneously reported that they were pleased to see that they were often quite accurate in estimating the BRs. And forth, inspecting the encoding of EEs upon presenting the actual BRs (i.e., parametric modulation of actual BR presentation by orthogonalized PR and EE) revealed that the activity in the bilateral ventral striatum was higher the smaller the EEs were (the more accurate the subjects were) regardless of their PR or desirability (i.e., for both good news and bad news; left striatum [-12 $14-6], 68$ voxel; right striatum [14 $12-6], 73$ voxel; $p<$ 0.05 , FWE-corr. at the peak level for the whole brain). The ventral striatum plays a central role in encoding positive prediction errors (Chase et al., 2015). In the context of a task in which subjects estimated BRs and were confronted with actual BRs that differed from their own estimates to a varying extent, greater accuracy corresponded to positive prediction error. Together with the debriefing self-reports, this finding supports the assumption that subjects focused on the degree of their accuracy during the presentation of BRs and thus were likely to reconsider their own risks subsequently at a segregated time point.

\section{DCM results}

After identifying the vmPFC as the valuation area in the context of belief updating, we applied DCM to test competing hypotheses about its causal role within the update circuit. First, we selected three nodes for the DCM based on the update-related group results revealed by the simplified categorical analysis (Fig. $3 C$, Table 3, contrasts $3 \mathrm{a}$ and $3 \mathrm{c}$ ). The first node was the dIPFC. This region was involved both in general updating (conjunction across all update categories; Fig. $3 C$ ), as well as in tracking errors in relevant prior beliefs (regarding $B R$ ) that were predictive of individual learning rates (Fig. $3 A$ ). Therefore, within the network activated by general updating, we chose the peak nearest to the learning rate-associated error tracking effect (MNI peak coordinate [ $\left[\begin{array}{lll}4 & 42 & 26\end{array}\right]$ ). To further ensure that the chosen dIPFC peak was indeed specifically recruited by updating, we contrasted the three E2 categories with E1 (second vs first self-risk estimation). In a separate group-level analysis with four contrast images ( $\mathrm{E} 2_{\text {unfavorable }}, \mathrm{E} 2_{\text {mid }}, \mathrm{E} 2_{\text {favorable }}$, and $\mathrm{E} 1$ ), we identified those regions that were more activated during updating beliefs about risks than during forming initial beliefs about risks (i.e., $\mathrm{E} 2_{\text {unfavorable }}, \mathrm{E} 2_{\text {mid }}$ and $\mathrm{E} 2_{\text {favorable }}>\mathrm{E} 1$, contrast $\left[\begin{array}{llll}1 & 1 & 1 & -3\end{array}\right]$ ). Note that E1 and E2 were otherwise comparable with respect to visual and motor requirements (Fig. 1). This additional analysis con- firmed that the dIPFC was significantly activated during belief updating relative to initial belief formation ( $p<0.05$, FWEcorrected at the peak level for the whole brain). Importantly, the dIPFC cluster overlapped with both the general updating conjunction effect and the error tracking effect. Given that the dlPFC is a crucial part of the working memory system for transient storage and manipulation of information (Eriksson et al., 2015), this region represents a key candidate for maintaining integrative information processing generally necessary for belief updating. We therefore refer to the dIPFC as the valence-independent "update processing" node. The second node was the vmPFC (MNI peak coordinate $\left.\left[\begin{array}{lll}-2 & 46 & -22\end{array}\right]\right)$. Its activity was greater in response to favorable than unfavorable updates and this valencecoding effect predicted the individual magnitudes of the optimism bias (Fig. $3 B, C$ ), thus forming a "valuation" node. Finally, we defined the dmPFC as our third node (MNI peak coordinate $\left[\begin{array}{lll}-16 & 44 & 40\end{array}\right]$ ). This region demonstrated a similar activity pattern as the vmPFC in the categorical GLM (Fig. $3 C$ ), but, in contrast to vmPFC, it has been associated with cognitive processes such as social inferences and perspective taking and less so with reward processing (Bzdok et al., 2013; de la Vega et al., 2016). Therefore, we will tentatively refer to the dmPFC as the "cognitive" node.

This simple architecture comprising three nodes allowed us to compare different models that implied either valence-guided or non-valence-guided explanations for the observed brain responses. Valence-guided explanations would be favored if the vmPFC were the source of both the valence-dependent filtering of the general update-processing signal and the subsequent influence on other prefrontal regions. Alternatively, non-valenceguided explanations would be supported if the vmPFC would receive a signal that is already modulated in a valence-dependent manner and have no driving influence on other prefrontal regions. Therefore, adopting a hypothesis driven approach, we limited our model space to 10 DCMs corresponding to these competing theories about the neural processing of belief updating (Fig. 4A). Although we could in principle construct a higher number of possible models, including more models would mainly obfuscate our analysis because additional alternative models would not be realistic (e.g., disconnected nodes) or be prone to overfitting but unable to provide a conclusive answer to our research question (e.g., valence modulates all connections).

In all 10 models, the event corresponding to the second risk estimation (E2, all three categories of updates) was specified as the exogenous input (Fig. 4A). This input entered the dlPFC (matrix C in DCM) because this region showed increased activity during all categories of belief updating (see the line chart in Fig. $3 C$ ). The models differed in their endogenous coupling (matrix A in DCM) such that $\mathrm{m} 1$ to $\mathrm{m} 5$ assumed a flow of neuronal states from dlPFC via vmPFC to dmPFC, whereas in models $\mathrm{m} 6$ to $\mathrm{m} 10$, the flow was from dlPFC via dmPFC to vmPFC. Given that we expected that the vmPFC would influence the dmPFC, models $\mathrm{m} 6$ to $\mathrm{m} 10$ represented null-hypotheses assuming the opposite course of influence. Furthermore, we systematically selected each of the possible coupling parameters to be the target of the valence-dependent modulation (matrix B in DCM; unfavorable updating, $\mathrm{U}$ or favorable updating, F). Given that we expected that favorable and unfavorable updating would differentially modulate the coupling in the network, $\mathrm{m} 5$ and $\mathrm{m} 10$ represented null hypotheses assuming no modulation at all. More specifically, we hypothesized that valence encoding would manifest through filtering of the incoming signal by the vmPFC and that the resulting differential valuation would further influence $\mathrm{dmPFC}$, as for- 

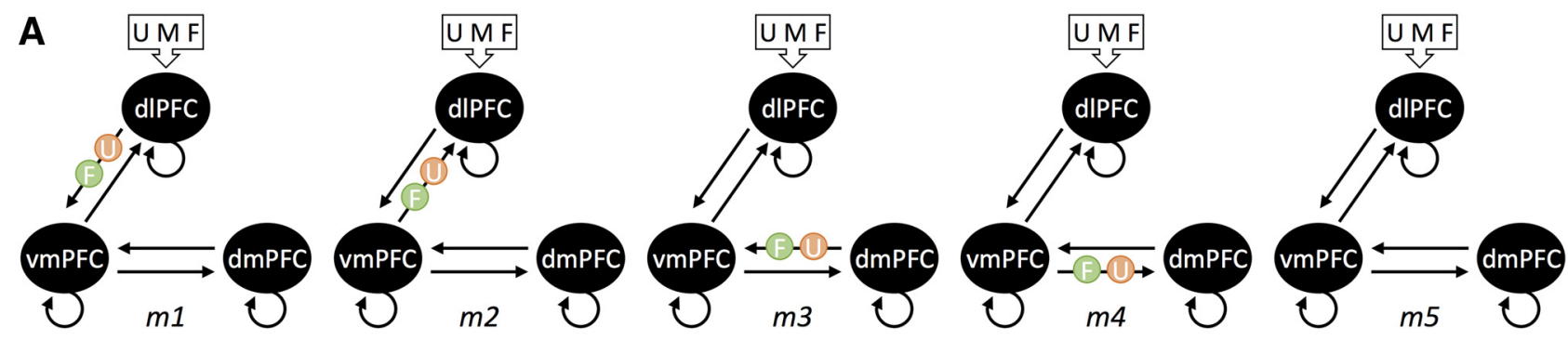

Contextual modulation: (1) Unfavorable updating, (F) Favorable updating
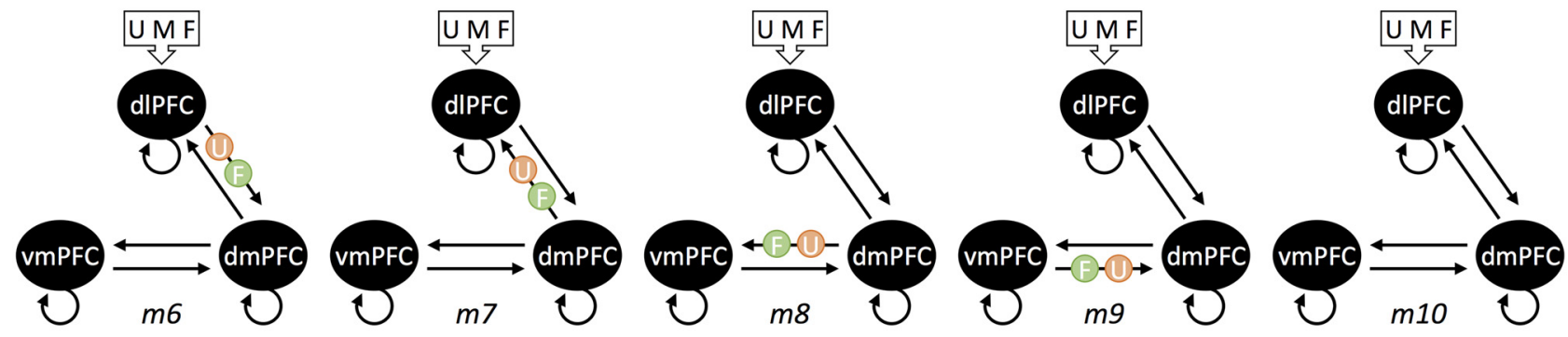

B

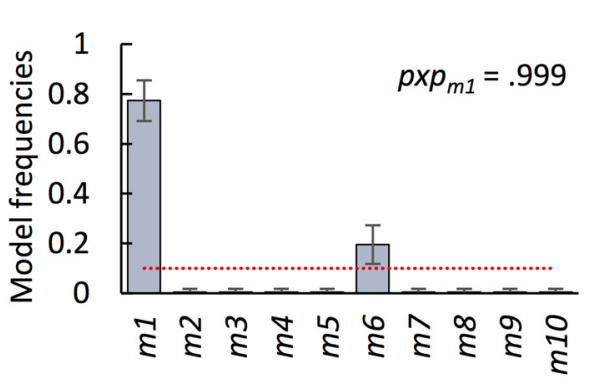

C
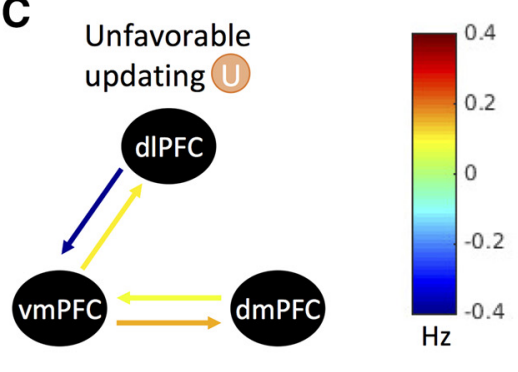

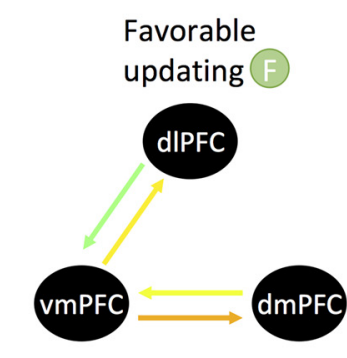

D

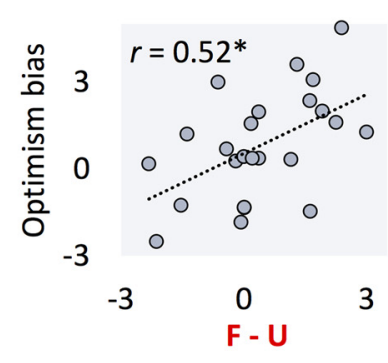

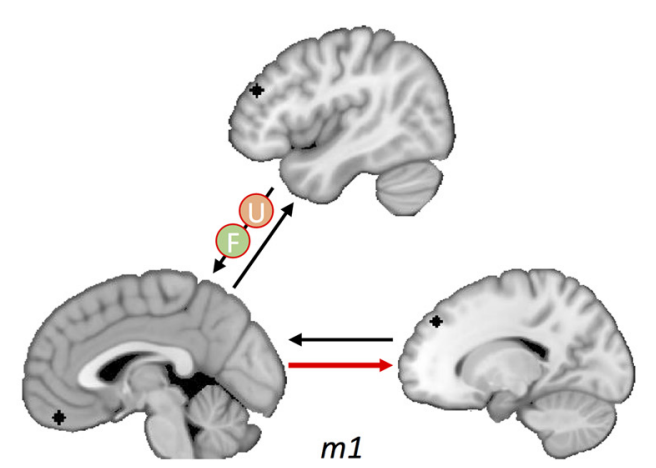

Figure 4. Neurocircuitry mechanisms underlying optimistic belief updating. $\boldsymbol{A}$, Ten different dynamic causal models varying in intrinsic connectivity and contextual modulation (unfavorable and favorable updating, $U$ and F) were specified. The model space encompassed three brain regions involved in updating: dIPFC, vmPFC, and dmPFC. $\boldsymbol{B}$, Bayesian model selection revealed that the model $\mathrm{m} 1$ best explained subjects' BOLD signal above and beyond chance (red dashed line). In this model, the coupling between dIPFC and vmPFC was differentially modulated by unfavorable and favorable updating. Therefore, the vmPFC filtered the incoming information in a valence-dependent manner and furthermore influenced the dmPFC. $C$, Connectivity parameters derived from $m 1$ show that the coupling between dIPFC and vmPFC tended to be weaker in the context of unfavorable relative to favorable updating. D, Optimism bias correlated with two parameters of 1 (highlighted in red): differential modulation of the dIPFC-vmPFC connection by favorable versus unfavorable updating (F-U) and the strength of the vmPFC-dmPFC connection (vmPFC::dmPFC). Therefore, subjects with a stronger optimism bias also demonstrated a greater valence-dependent filtering of incoming information by vmPFC and a greater transmission of this differential signal further to dmPFC.

malized in $\mathrm{m} 1$. Alternatively, the valence-dependent modulation could have affected one of the other couplings (e.g., from dlPFC to $\mathrm{dmPFC}$ as formalized in $\mathrm{m} 6$ ). In these cases, the filtering of the incoming signal would not be attributed to vmPFC and/or there would be no primary influence of the vmPFC on dmPFC. All models except of $\mathrm{m} 5$ and $\mathrm{m} 10$ were equally complex but differed with respect to the flow of neuronal states and the coupling, which was subject to contextual modulation, allowing for evidence-based hypothesis testing.
Bayesian model comparison confirmed that the model $\mathrm{m} 1$ had the greatest evidence, above and beyond chance, $E f=0.77$, pxp $=0.999$ (Fig. 4B). The selected model assumed a cyclic signal flow from the dlPFC via vmPFC to dmPFC and a valencedependent modulation of the coupling from dIPFC to vmPFC. The low evidence of models without modulations ( $\mathrm{m} 5$ and $\mathrm{m} 10$ ) indicates that the valence-dependent modulation of effective connectivity was indeed necessary to adequately predict subjects' network activity. Furthermore, of the eight models with modu- 
Table 4. DCM parameter estimates of the model $\mathrm{m} 1$ and correlations with measures of optimism bias

\begin{tabular}{lcllllll}
\hline \multicolumn{7}{c}{$r$, optimism } \\
& M (SD) & $p, t$ test & bias & $p$ & $r$, asymmetry & $p$ \\
\hline Matrix A & & & & & & \\
dl::dl & $-0.01(0.09)$ & $0.000^{*}$ & -0.19 & 0.361 & -0.21 & 0.323 \\
dl:::vm & $-0.06(0.12)$ & 0.030 & -0.23 & 0.277 & -0.05 & 0.819 \\
vm:::dl & $0.10(0.15)$ & $0.000^{*}$ & -0.09 & 0.679 & -0.15 & 0.485 \\
vm::vm & $-0.07(0.09)$ & $0.000^{*}$ & -0.20 & 0.348 & -0.20 & 0.342 \\
vm:::dm & $0.15(0.14)$ & $0.000^{*}$ & 0.47 & $0.020^{*}$ & 0.49 & 0.015 \\
dm:::vm & $0.08(0.124)$ & $0.004^{*}$ & 0.27 & 0.207 & 0.32 & 0.127 \\
dm::dm & $-0.02(0.03)$ & $0.001^{*}$ & -0.49 & 0.015 & -0.56 & 0.005 \\
Matrix B & & & & & & \\
U on dl:::vm & $-0.35(1.06)$ & 0.123 & -0.23 & 0.275 & -0.31 & 0.147 \\
F on dl::vm & $0.05(1.00)$ & 0.822 & 0.49 & 0.015 & 0.36 & 0.088 \\
F- U & $0.39(1.42)$ & 0.188 & 0.52 & $0.009^{*}$ & 0.48 & 0.018 \\
Matrix C & & & & & & \\
U M F to dl & $0.12(0.07)$ & 0.000 & 0.20 & 0.340 & 0.13 & 0.541 \\
\hline
\end{tabular}

Parameter estimates are shown in Hertz, self-connections were log-transformed.

$\mathrm{dl}$, Dorsolateral prefrontal cortex; vm, ventromedial prefrontal cortex; dm, dorsomedial prefrontal cortex; "::," endogenous connection; $U$, unfavorable updating; $M$, mid-updating; $F$, favorable updating.

${ }^{*}$ Equivalent to $p<0.05$, Bonferroni-corrected for multiple comparisons (Matrix A, $t$ test, $p<0.007$ corrected for 7 comparisons; $r$, optimism bias, $p<0.025$ corrected for 2 comparisons with a priori hypotheses).

lations, $\mathrm{m} 1$ still had greater evidence than $\mathrm{m} 6$ and other alternative models. This finding supports the hypothesis that the vmPFC filtered the incoming signal in a valence-dependent manner and influenced the dmPFC.

Third, we further inspected and analyzed the parameter estimates derived from the winning model $\mathrm{m} 1$. We hypothesized that the magnitude of valence-dependent modulation (the difference between F and U, F-U) of the dlPFC-vmPFC coupling would correlate with the optimism bias across subjects because, the stronger this modulation, the greater should be the response of the vmPFC to different valences of updating. Furthermore, we expected that the strength of the connection from vmPFC toward dmPFC would also correlate with the optimism bias, assuming that this coupling represents the influence of valuation on ongoing cognitive processing. Modulation parameters and coupling patterns in the context of favorable and unfavorable updating are reported in Table 4 and plotted in Figure 4C. On average, the coupling from dlPFC to vmPFC decreased in the context of unfavorable updating relative to favorable updating. However, this difference did not reach significance due to the large variance of modulation estimates for $\mathrm{U}$ and $\mathrm{F}$. It is of greater importance though that the valence-dependent modulation of the dlPFCvmPFC coupling (the difference between $\mathrm{F}$ and U, F-U) correlated with the size of the optimism bias across subjects (Fig. 4D). This relationship explains how the data observed in the fMRI analysis were caused. Subjects with a greater optimism bias had a stronger valence-dependent filtering by vmPFC, resulting in an increased BOLD response to favorable than unfavorable updating in vmPFC. Moreover, the individual strength of the coupling from vmPFC to $\mathrm{dmPFC}$ also correlated with optimism bias (both correlations corrected for multiple comparisons). Therefore, the stronger the optimism bias, the stronger was the influence of valuation on ongoing cognitive processing, mediated by the coupling from vmPFC to $\mathrm{dmPFC}$. In addition, the inspection of all possible correlation coefficients (Table 4) revealed that the endogenous self-connection of the dmPFC inversely correlated with the optimism bias (not corrected for multiple comparisons). Therefore, the stronger the optimism bias, the weaker the self-inhibition of the dmPFC. Together, these parameter estimates indicate a self-enhancing cyclic flow between vmPFC and dmPFC. In subjects with high optimism bias, the
vmPFC filtered the incoming information dependent on valence. This differential signal was then forwarded to the $\mathrm{dmPFC}$ and there enhanced by the reduced self-inhibition.

\section{Discussion}

The present study provides converging evidence that the value of desirable beliefs can influence ongoing cognitive processing. Participants demonstrated an optimism bias because they were more likely to update beliefs regarding their risks in response to good news than bad news (learning that BRs of the risks were lower vs higher than expected). This finding was also confirmed by computational modeling that formally controlled for valence-unrelated variables that influence updating (Shah et al., 2016; Kuzmanovic and Rigoux, 2017; EE and PR of the new information). Given that we ruled out these alternative, valence-independent explanations, manipulated the desirability of the new information independently of prior beliefs, and demonstrated that the optimism bias was unrelated to the size of risk estimates or BRs, we conclude that information integration was indeed biased by the motivation to adopt the most favorable beliefs about one's future.

Furthermore, fMRI results showed that the vmPFC tracked the value of updating. In the context of good news, large updates toward lower risk estimates improve the ultimate risk perception, but after bad news, large updates toward higher risk estimates worsen the ultimate belief. In turn, small updates (small or no change in beliefs) also acquire opposing values in the context of good and bad news, respectively. Although small updates after good news are unfavorable because they disregard the opportunity to improve risk estimates, small updates after bad news are favorable because they prevent worsening of risk estimates. The activity pattern in the vmPFC showed exactly this pattern: it increased with increasing updates toward lower risks (after good news) and with decreasing updates after bad news. Therefore, not only improving beliefs, but also avoiding the worsening of beliefs triggered the vmPFC activity. Previous studies on optimism bias that included risk estimates for self and a similar other person already indicated a positive value of avoiding threatening belief updates by disregarding undesirable new information. Here, particularly the decreased updating in self-related trials with bad news (relative to a comparably high amount of updating in selfrelated trials with good news and all other-related trials) was driving the optimism bias (Kuzmanovic et al., 2015, 2016a). Furthermore, research on context dependency of option values has shown that both gaining a reward (i.e., improving the current state, e.g., change from $1 \$$ to $2 \$$ ) and knowingly avoiding punishment (i.e., current state is unchanged, e.g., $1 \$$, but the possible loss, e.g., $-1 \$$, is avoided) acquired a positive value and were tracked by the vmPFC (Palminteri et al., 2015). Lesion studies (Camille et al., 2011) and meta-analyses (Yarkoni et al., 2011; Diekhof et al., 2012; Levy and Glimcher, 2012; Bartra et al., 2013; Clithero and Rangel, 2014; Chase et al., 2015) have consistently shown that vmPFC is associated with valuation of rewards. In light of this literature, our results highlight that not only external rewards such as food or money, but also intrinsic rewards such as favorable beliefs, recruit the same valuation system. Moreover, the vmPFC was shown to automatically encode the value of objects (faces, houses, and paintings) independently of the explicit task instruction (Lebreton et al., 2009). Consistent with this automatic valuation and studies demonstrating unconscious motivational influences (Pessiglione et al., 2007), debriefing in our study revealed that subjects were unaware of their valencedependent updating indicating that the valuation of belief updates need not require a voluntary process. 
Extending previous work (Kuzmanovic et al., 2016a), the tracking of valence by vmPFC could be uniquely attributed to update sizes above and beyond the influence of EEs, PR of errors, actual BRs, or final risk estimates. In other words, valence of updating depended on improvement or worsening of final beliefs relative to initial beliefs regardless of the beliefs or the new information per se. Moreover, we hypothesized that the valencetracking effect in the vmPFC would be more pronounced in subjects with greater optimism bias because belief formation should be biased by the desire to make favorable updates only if favorable updates also have a positive value. Whereas favorable future outlooks are likely to be experienced as pleasant, the sensitivity to the value of such prospects may differ among individuals depending on their current state or their personality. Indeed, subjects with a stronger optimism bias exhibited a greater valencetracking effect in the vmPFC, confirming that the vmPFC activity is sensitive to the subjective value of stimuli (Grabenhorst and Rolls, 2011; Winecoff et al., 2013).

But what was the mechanism underlying this valencedependent recruitment of the vmPFC that was able to influence ongoing belief formation? One possibility is that, in the context of favorable (relative to unfavorable) updating, the vmPFC amplified incoming signals and further influenced other prefrontal regions. Alternatively, the vmPFC may be influenced by other prefrontal regions, the activity of which has already been modulated in a valence-dependent manner. We tested these competing hypotheses by comparing dynamic causal models comprising regions differentially recruited during favorable and unfavorable updating. The models consisted of three nodes with distinct functional signatures: the dIPFC represented an "update processing" node that received the exogenous input, the vmPFC was included as the "valuation" node, and the dmPFC represented a "cognitive" node. The dlPFC was a part of an extended network that was generally involved in updating (both favorable and unfavorable). The same region was also engaged in tracking errors in BR estimates, whereas the strength of this tracking was predictive of individual learning rates. In contrast, both vmPFC and dmPFC showed greater activity for favorable than unfavorable updating. Having demonstrated that the vmPFC tracked the valence of belief updating in a strictly controlled, task-related manner, we use the label "cognitive" node for the dmPFC to distinguish it from the valuation-related vmPFC. Although we cannot specify the exact kind of cognitive processing associated with the dmPFC recruitment, recent meta-analyses indicate clear functional dissociations with vmPFC being selectively associated with reward-related tasks and dmPFC being preferentially involved in self-referential cognitive processes such as social inferences and perspective taking (Bzdok et al., 2013; de la Vega et al., 2016). In the context of reconsidering one's own risk with respect to that of others, social inferences and perspective taking seem highly plausible and their valenceguided use may provide the means of arriving at a particular, preferred conclusion (Kunda, 1990; Shepperd et al., 2002).

The Bayesian model comparison identified an optimum dynamic causal model that had a reciprocal information flow from dIPFC via vmPFC to dmPFC, with a valence-dependent modulation of the coupling from dIPFC to vmPFC. This shows that particularly the vmPFC filtered the incoming signal in a valencedependent manner and influenced the dmPFC accordingly. Importantly, both of these circuit features predicted individual magnitudes of the optimism bias. Subjects with a stronger optimism bias showed a greater increase in the dIPFC-vmPFC coupling during favorable (relative to unfavorable) updating. Moreover, biased belief updating was greater the stronger the transmission of this valence-dependent signal from vmPFC to dmPFC. Therefore, the magnitude of the influence of valuation on ongoing cognitive processing, mediated by the coupling from vmPFC to dmPFC, predicted how much participants were biased toward more favorable updates. This finding complies with previous studies on functional connectivity, where the increase in connection between vmPFC ([3 $51-16])$ and dmPFC ([ -1556 37]) predicted greater context-initiated reevaluation of choice options across subjects (Rudorf and Hare, 2014).

Previous studies on effective connectivity identified the vmPFC as a target of the directional influence of other regions. The coupling from hippocampus to vmPFC was increased when people chose better remembered options (Gluth et al., 2015). Furthermore, the coupling from dlPFC to vmPFC was increased during decisions to resist tempting short-term rewards and to choose greater, but delayed rewards instead, and this effect was predictive of between-subject differences in delay discounting (Hare et al., 2014). These and our findings share the general idea of dynamic reciprocal influences between the valuation system and other cognitive systems. However, our study is the first to show the opposite direction of influence, namely the influence of the vmPFC on the dmPFC that mediates value-guided belief formation.

In the resulting mechanistic model of belief formation, the valuation of ongoing conclusions influences further cognitive processing, which in turn determines the final belief. Therefore, our results provide novel evidence for the notion that motivation to maximize pleasant beliefs reinforces those cognitive processes that are most likely to yield favorable perspectives. Leaving no possibility of reinterpretation of the observed effects in entirely valence-independent terms, we substantially contribute to resolving the still persisting "hot versus cold cognition" controversy (Kunda, 1990). As soon as we have a preference for one conclusion over another, we may be in danger of automatically adjusting the knowledge that we recall and the inferential rules that we apply in such a way as to support the preferred conclusion. This bias in our reasoning has far-reaching implications for diverse decisions that we make in our everyday lives, whether in private or in professional contexts. Although it can serve to protect us from discouraging and gloomy beliefs, it may also promote risk underestimations and discriminating judgments.

\section{References}

Andersson JL, Skare S, Ashburner J (2003) How to correct susceptibility distortions in spin-echo echo-planar images: application to diffusion tensor imaging. Neuroimage 20:870-888. CrossRef Medline

Bartra O, McGuire JT, Kable JW (2013) The valuation system: a coordinatebased meta-analysis of BOLD fMRI experiments examining neural correlates of subjective value. Neuroimage 76:412-427. CrossRef Medline

Bastin J, Deman P, David O, Gueguen M, Benis D, Minotti L, Hoffman D, Combrisson E, Kujala J, Perrone-Bertolotti M, Kahane P, Lachaux JP, Jerbi K (2017) Direct recordings from human anterior insula reveal its leading role within the error-monitoring network. Cereb Cortex 27:15451557. CrossRef Medline

Bzdok D, Langner R, Schilbach L, Engemann DA, Laird AR, Fox PT, Eickhoff SB (2013) Segregation of the human medial prefrontal cortex in social cognition. Front Hum Neurosci 7:232. CrossRef Medline

Camille N, Griffiths CA, Vo K, Fellows LK, Kable JW (2011) Ventromedial frontal lobe damage disrupts value maximization in humans. J Neurosci 31:7527-7532. CrossRef Medline

Chase HW, Kumar P, Eickhoff SB, Dombrovski AY (2015) Reinforcement learning models and their neural correlates: an activation likelihood estimation meta-analysis. Cogn Affect Behav Neurosci 15:435-459. CrossRef Medline

Clithero JA, Rangel A (2014) Informatic parcellation of the network involved in the computation of subjective value. Soc Cogn Affect Neurosci 9:1289-1302. CrossRef Medline 
Cumming G (2014) The new statistics: why and how. Psychol Sci 25:7-29. CrossRef Medline

Daunizeau J, Adam V, Rigoux L (2014) VBA: a probabilistic treatment of nonlinear models for neurobiological and behavioural data. PLoS Comput Biol 10:e1003441. CrossRef Medline

de la Vega A, Chang LJ, Banich MT, Wager TD, Yarkoni T (2016) Largescale meta-analysis of human medial frontal cortex reveals tripartite functional organization. J Neurosci 36:6553-6562. CrossRef Medline

Diekhof EK, Kaps L, Falkai P, Gruber O (2012) The role of the human ventral striatum and the medial orbitofrontal cortex in the representation of reward magnitude - an activation likelihood estimation meta-analysis of neuroimaging studies of passive reward expectancy and outcome processing. Neuropsychologia 50:1252-1266. CrossRef Medline

Eil D, Rao JM (2011) The good news-bad news effect: asymmetric processing of objective information about yourself. Am Econ J Microecon 3:114138. CrossRef

Eriksson J, Vogel EK, Lansner A, Bergström F, Nyberg L (2015) Neurocognitive architecture of working memory. Neuron 88:33-46. CrossRef Medline

Fouragnan E, Queirazza F, Retzler C, Mullinger KJ, Philiastides MG (2017) Spatiotemporal neural characterization of prediction error valence and surprise during reward learning in humans. Sci Rep 7:4762. CrossRef Medline

Friston KJ (2011) Functional and effective connectivity: a review. Brain Connect 1:13-36. CrossRef Medline

Garrett N, Sharot T (2017) Optimistic update bias holds firm: three tests of robustness following Shah et al. Conscious Cogn 50:12-22. CrossRef Medline

Garrett N, Sharot T, Faulkner P, Korn CW, Roiser JP, Dolan RJ (2014) Losing the rose tinted glasses: neural substrates of unbiased belief updating in depression. Front Hum Neurosci 8:639. CrossRef Medline

Gluth S, Sommer T, Rieskamp J, Büchel C (2015) Effective connectivity between hippocampus and ventromedial prefrontal cortex controls preferential choices from memory. Neuron 86:1078-1090. CrossRef Medline

Grabenhorst F, Rolls ET (2011) Value, pleasure and choice in the ventral prefrontal cortex. Trends Cogn Sci 15:56-67. CrossRef Medline

Hare TA, Hakimi S, Rangel A (2014) Activity in dlPFC and its effective connectivity to vmPFC are associated with temporal discounting. Front Neurosci 8:50. CrossRef Medline

Hughes BL, Zaki J (2015) The neuroscience of motivated cognition. Trends Cogn Sci 19:62-64. CrossRef Medline

Klein TA, Ullsperger M, Danielmeier C (2013) Error awareness and the insula: links to neurological and psychiatric diseases. Front Hum Neurosci 7:14. CrossRef Medline

Kolling N, Behrens T, Wittmann MK, Rushworth M (2016) Multiple signals in anterior cingulate cortex. Curr Opin Neurobiol 37:36-43. CrossRef Medline

Korn CW, Prehn K, Park SQ, Walter H, Heekeren HR (2012) Positively biased processing of self-relevant social feedback. J Neurosci 32:1683216844. CrossRef Medline

Korn CW, Sharot T, Walter H, Heekeren HR, Dolan RJ (2014) Depression is related to an absence of optimistically biased belief updating about future life events. Psychol Med 44:579-592. CrossRef Medline

Kunda Z (1990) The case for motivated reasoning. Psychol Bull 108:480498. CrossRef Medline

Kuzmanovic B, Rigoux L (2017) Valence-dependent belief updating: computational validation. Front Psychol 8:1087. CrossRef Medline

Kuzmanovic B, Jefferson A, Vogeley K (2015) Self-specific optimism bias in belief updating is associated with high trait optimism. Journal of Behavioral Decision Making 28:281-293. CrossRef

Kuzmanovic B, Jefferson A, Vogeley K (2016a) The role of the neural reward circuitry in self-referential optimistic belief updates. Neuroimage 133: 151-162. CrossRef Medline

Kuzmanovic B, Rigoux L, Vogeley K (2016b) Brief report: reduced optimism bias in self-referential belief updating in high-functioning autism. J Autism Dev Disord. Advance online publication. Retrieved October 18, 2016. doi:org/10.1007/s10803-016-2940-0.

Lebreton M, Jorge S, Michel V, Thirion B, Pessiglione M (2009) An automatic valuation system in the human brain: evidence from functional neuroimaging. Neuron 64:431-439. CrossRef Medline

Lefebvre G, Lebreton M, Meyniel F, Bourgeois-Gironde S, Palminteri S (2017) Behavioural and neural characterization of optimistic reinforce- ment learning. Nature Human Behaviour. Advance online publication. Retrieved March 20, 2017. doi:org/10.1038/s41562-017-0067.

Levy DJ, Glimcher PW (2012) The root of all value: a neural common currency for choice. Curr Opin Neurobiol 22:1027-1038. CrossRef Medline Mumford JA, Poline JB, Poldrack RA (2015) Orthogonalization of regressors in FMRI models. PLoS One 10:e0126255. CrossRef Medline

Palminteri S, Khamassi M, Joffily M, Coricelli G (2015) Contextual modulation of value signals in reward and punishment learning. Nat Commun 6:8096. CrossRef Medline

Palminteri S, Lefebvre G, Kilford EJ, Blakemore SJ (2017) Confirmation bias in human reinforcement learning: evidence from counterfactual feedback processing. PLoS Comput Biol 13:e1005684. CrossRef Medline

Penny WD (2012) Comparing dynamic causal models using AIC, BIC and free energy. Neuroimage 59:319-330. CrossRef Medline

Pessiglione M, Schmidt L, Draganski B, Kalisch R, Lau H, Dolan RJ, Frith CD (2007) How the brain translates money into force: a neuroimaging study of subliminal motivation. Science 316:904-906. CrossRef Medline

Power JD, Barnes KA, Snyder AZ, Schlaggar BL, Petersen SE (2012) Spurious but systematic correlations in functional connectivity MRI networks arise from subject motion. Neuroimage 59:2142-2154. CrossRef Medline

Rigoux L, Stephan KE, Friston KJ, Daunizeau J (2014) Bayesian model selection for group studies revisited. Neuroimage 84:971-985. CrossRef Medline

Roese NJ, Olson JM (2007) Better, stronger, faster self-serving judgment, affect regulation, and the optimal vigilance hypothesis. Perspect Psychol Sci 2:124-141. CrossRef Medline

Rudman LA, Dohn MC, Fairchild K (2007) Implicit self-esteem compensation: automatic threat defense. Journal of Personality and Social Psychology 93:798-813. CrossRef Medline

Rudorf S, Hare TA (2014) Interactions between dorsolateral and ventromedial prefrontal cortex underlie context-dependent stimulus valuation in goal-directed choice. J Neurosci 34:15988-15996. CrossRef Medline

Shah P, Harris AJ, Bird G, Catmur C, Hahn U (2016) A pessimistic view of optimistic belief updating. Cogn Psychol 90:71-127. CrossRef Medline

Sharot T, Garrett N (2016) Forming beliefs: why valence matters. Trends Cogn Sci 20:25-33. CrossRef Medline

Sharot T, Guitart-Masip M, Korn CW, Chowdhury R, Dolan RJ (2012) How dopamine enhances an optimism bias in humans. Curr Biol 22: 1477-1481. CrossRef Medline

Sharot T, Korn CW, Dolan RJ (2011) How unrealistic optimism is maintained in the face of reality. Nat Neurosci 14:1475-1479. CrossRef Medline

Shenhav A, Straccia MA, Cohen JD, Botvinick MM (2014) Anterior cingulate engagement in a foraging context reflects choice difficulty, not foraging value. Nat Neurosci 17:1249-1254. CrossRef Medline

Shepperd JA, Carroll P, Grace J, Terry M (2002) Exploring the causes of comparative optimism. Psychologica Belgica 42:65-98.

Smith SM, Jenkinson M, Woolrich MW, Beckmann CF, Behrens TE, Johansen-Berg H, Bannister PR, De Luca M, Drobnjak I, Flitney DE, Niazy RK, Saunders J, Vickers J, Zhang Y, De Stefano N, Brady JM, Matthews PM (2004) Advances in functional and structural MR image analysis and implementation as FSL. Neuroimage 23:S208-S219. CrossRef Medline

Stephan KE, Penny WD, Moran RJ, den Ouden HE, Daunizeau J, Friston KJ (2010) Ten simple rules for dynamic causal modeling. Neuroimage 49: 3099-3109. CrossRef Medline

Tesser A (2000) On the confluence of self-esteem maintenance mechanisms. Personality and Social Psychology Review 4:290-299. CrossRef

Wessel JR, Danielmeier C, Morton JB, Ullsperger M (2012) Surprise and error: common neuronal architecture for the processing of errors and novelty. J Neurosci 32:7528-7537. CrossRef Medline

Winecoff A, Clithero JA, Carter RM, Bergman SR, Wang L, Huettel SA (2013) Ventromedial prefrontal cortex encodes emotional value. J Neurosci 33:11032-11039. CrossRef Medline

Xu J, Moeller S, Auerbach EJ, Strupp J, Smith SM, Feinberg DA, Yacoub E, Uğurbil K (2013) Evaluation of slice accelerations using multiband echo planar imaging at 3 T. Neuroimage 83:991-1001. CrossRef Medline

Yarkoni T, Poldrack RA, Nichols TE, Van Essen DC, Wager TD (2011) Large-scale automated synthesis of human functional neuroimaging data. Nat Methods 8:665-670. CrossRef Medline 